\title{
A Scale of Parental Anxiety about Pediatric Emergency Medical Care Services of Japan: Development, Reliability, Validity, Generalizability and Usefulness
}

\author{
Ikuko Sobue ${ }^{1}$, Kimie Tanimoto², Susumu Itoh ${ }^{3}$ \\ ${ }^{1}$ Division of Nursing Science, Graduate School of Biomedical \& Health Sciences, Hiroshima University, Hiroshima, Japan \\ ${ }^{2}$ Division of Nursing Science, Faculty of Medicine, Kagawa University, Kagawa, Japan \\ ${ }^{3}$ Division of Medical Science, Faculty of Medicine, Kagawa University, Kagawa, Japan \\ Email:sobue@hiroshima-u.ac.jp
}

How to cite this paper: Sobue, I., Tanimoto, K. and Itoh, S. (2017) A Scale of Parental Anxiety about Pediatric Emergency Medical Care Services of Japan: Development, Reliability, Validity, Generalizability and Usefulness. Health, 9, 1427-1458.

https://doi.org/10.4236/health.2017.910105

Received: April 15, 2017

Accepted: September 27, 2017

Published: September 30, 2017

Copyright (c) 2017 by authors and Scientific Research Publishing Inc. This work is licensed under the Creative Commons Attribution International License (CC BY 4.0).

http://creativecommons.org/licenses/by/4.0/

\section{(c) (i) Open Access}

\begin{abstract}
Aim: The purpose of this study was to develop a scale, "parental anxiety about pediatric emergency medical care services" (PAPEMCS), and to evaluate its psychometric properties. Methods: Participants were 14,510 parents with children 6 years old or younger in Kagawa Prefecture. Using each half of the participants, exploratory factor analysis was performed to generate items and factors for the PAPEMCS, and confirmatory factor analysis (CFA) was used to establish the construct validity. The generalizability of the PAPEMCS was evaluated by congruence tests and multigroup CFA. The usefulness of the PAPEMCS was established by the relationship between the PAPEMCS and non-urgent usage of pediatric emergency medical care services (PEMCS). Results: The PAPEMCS compromised 4 factors: "anxiety about quality of PEMCS", "anxiety about PEMCS system", "anxiety about public support", and "anxiety about private support". All reliability estimates (polychoric ordinal alpha coefficients, item-rest correlations), the item discrimination, 5 fit indices for CFA, the convergent validity (indicator reliabilities, composite reliabilities, average variance extracteds), and the discriminant validity fulfilled the acceptability thresholds. All generalizability estimates fulfilled the predetermined levels of acceptability (Tucker's congruence coefficients, congruence tests, strict factorial invariance). The usefulness of the PAPEMCS was established by the higher scores of the PAPEMCS being related to non-urgent usage of PEMCS. Conclusions: The PAPEMCS demonstrated satisfactory reliability, validity, generalizability and usefulness. The PAPEMCS is useful to quantify the contents and extent of parental anxiety about PEMCS, and to cla-
\end{abstract}


rify the mechanisms of non-urgent PEMCS usage.

\section{Keywords}

Parental Anxiety, Pediatric Emergency Medical Care Services, Reliability, Validity, Generalizability and Usefulness, Scale Development

\section{Introduction}

The appropriate enforcement of pediatric emergency medical care service (PEMCS) is a vital component of pediatric health services. However, around the world, especially in developed nations, pediatric emergency department (PED) crowding due to PED visits for non-urgent health concerns prevents the efficient and efficacious use of health services. The overcrowding threatens the quality (treatment delays for children requiring attention and urgent medical care), wastes resources, and is a financial burden to parents and to society [1]-[6]. Also, it increases a lack of continuity care and adequate access to primary care [7] [8]. In order to enhance the wellness of children and family, it is necessary to not only reduce crowded situations by improving the pediatric emergency systems (triage, human resources, equipment), but also to examine the causes of non-urgent usage. The characteristics of children who returned to PED within 72 hours $(6968,4.3 \%)$ and the characteristics of children who were admitted to the hospital on their return visit $(2925,42 \%)$ would help in directing future strategies to reduce potentially avoidable reattendance and admission [9].

A lot of previous studies have demonstrated that non-urgent PED users are characterized by high levels of social, educational, and financial disadvantages, and include parents with only one child, young mothers, single parents, and parents with a low education and low income that receive public insurance or have no insurance [7] [10] [11] [12] [13]. Non-urgent PED users are inferred as people who do not adhere to appropriate PED usage because of their educational and socioeconomic vulnerability, low health literacy and/or shortage of child care experience [14]-[22].

Regarding the reasons of PED usage, parents, especially those engaging in non-urgent PED usage, have the sense of anxiety over child's illness [20] [21] [22] [23] [24]. Parents want to rule out risks, receive early treatment, and obtain relief, regardless of whether their child actually needs a PED [25] [26] [27] [28]. Parents prefer a PED due to anxieties about accessing higher quality medical care, including the availability of such services as laboratory tests, hospitalization and family-centered care [5] [23] [25] [28] [29] [30], and of short waiting times [10].

In addition, parents are full of anxiety about PEMCS systems, such as convenience of 24-hour accessibility [5] [23] [29]. Health care policy in Japan consolidates medical care resources and expands medical regions in order to provide 
24-hour PEMCS [31] [32]. However, all local governments cannot provide 24-hour PEMCS [33]. The emergency medical care system in Japan has developed through three services: primary emergency care for mildly ill patients who can be treated on an out-patient basis (holiday emergency clinics, night emergency centers, etc.) in 378 pediatric care regions [34], secondary emergency care for moderate-to-severe patients requiring hospitalization in 143 secondary care regions [35], tertiary emergency care for all patients including children whose lives are at risk, and the government intention is to have one emergency and critical care center per one million people [36] [37]. Because PEMCS systems differ multifariously in medical regions depending on limited health resources, such as only night emergency clinic or a group of hospitals on rotation duty as secondary emergency care, parents tend to seek and use PEDs with a sense of excessive anxiety about PEMCS [38] [39] [40] [41]. When parents feel there is less access to health services, their anxiety about a child's illness increases [20] [21]. As a result of anxiety about quality or access of PED, parents appear to visit PED for children with non-urgent condition.

However, no studies have been conducted to clearly explain what aspects of parental vulnerability or PEMCS systems are related to what kind of anxiety about PEMCS [22] [42]. The suitability of a PEMCS system is not evaluated from the viewpoint of the users residing in the medical region. In terms of reasons, it should be particularly pointed out that the socioeconomic vulnerabilities of non-urgent PED users have quantitatively been examined [7] [10] [11] [12] [13], and the anxiety about or reasons for PEMCS usage have qualitatively been examined by interviewing dozens of parents [5] [26] [29] [38] [39] [40] [41]. In sum, the mechanisms of multifarious risks causing non-urgent PED usage remain unknown, and the appropriate PEMCS to address the risks remain unknown.

To provide suitable PEMCS that eliminate parental anxieties with limited health resources, it is firstly essential to investigate the multifarious parental anxieties about PEMCS. If a scale for quantifying the contents and extent of multifarious anxieties about PEMCS could measure specific anxieties caused by specific risks, as well as comprehensive anxieties integrating individual anxieties, the scale would be useful to examine multifarious risks of PEMCS. This study aims to develop a scale to quantify the contents and extent of parental anxiety about PEMCS, and to examine the reliability, validity, generalizability and usefulness of the scale.

\section{Methods}

\subsection{Participants}

In order to ensure the generalization of the scale, Kagawa Prefecture was selected as an area that fulfills the research requirements: multifarious family structure, PEMCS systems, and diverse geographical features. The population of Kagawa Prefecture is 995,842 [43] and its area is $1876.51 \mathrm{~km}^{2}$, with 24 po- 
pulated islands [44].

The PEMCS systems in Kagawa Prefecture represent multiple PEMCS available in Japan. For example, the PEMCS system of the Takamatsu secondary medical region (Takamatsu) is constituted of typical systems of PEMCS in Japan: primary (a pediatric emergency night clinic), secondary (a group of hospitals on rotational duty) and tertiary (Kagawa University Hospital) [38] [45]. Okawa or Mitoyo secondary medical region (Mitoyo) care is performed with a PEMCS system of a pediatric emergency night clinic only, and Shozu secondary medical region (Shozu), a remote island, care is performed with an on-call emergency medical service system at two hospitals. These systems represent the unimproved PEMCS system similar to other secondary medical regions in Japan. In Chusan secondary medical region (Chusan), a pediatric hospital performs PEMCS in the same way as an emergency department in the U.S. (primary, secondary, and tertiary; 24 hours a day, 365 days a year). In addition, Kagawa Medical Association administers a holiday on-duty doctor system at clinics and hospitals for all residents, and publicizes this service in newspapers, public information magazines, and on a website.

Although the PEMCS system in Chusan is rare in Japan, it is important in the future development of PEMCS to examine not only other PEMCS systems, but also the PEMCS system itself. Therefore, as an area to ensure the generalization of the scale, Kagawa Prefecture was selected.

The inclusion criteria were as follows: the family who has a child six years old or younger because many of such children visit PED [46] [47] [48] [49] and the family living in all areas of Kagawa Prefecture because of its multifarious family structure, PEMCS systems, and diverse geographical features. The medical expenses of this age were free [50]. Exclusion criterion was the family who only had children seven years old or older.

In order to fulfill the inclusion criteria, the selected participants were parents of children attending kindergartens or nursery schools, or those undergoing a health examination at a health center (one and a half years old or three years old) in Kagawa Prefecture.

\subsection{Questionnaire Item Development}

First, based on literature review, three pediatric and four community nursing researchers developed 43 questionnaire items that depict parental anxiety about PEMCS: information, system, or quality of PEMCS; public support; private support; and parental care ability [5] [25] [29] [51] [52] [53] [54]. To measure the intensity of parental anxiety regarding PEMCS when a child became ill, each PEMCS item was constituted of a four-point response option, ranging from " $1=$ no anxiety" to " $4=$ high anxiety". Second, twelve pediatricians examined the appropriateness of expressions and adequacy of the contents of the items.

Third, the preliminary research was performed for parents who only had children of seven years old or older, because the main research was intended to 
secure families with children of six years old or younger as much as possible. In addition, if both the preliminary research and the main research participants are parents whose children are the same age, when the investigation institutions handed a questionnaire to parents, it was considered to be difficult to exclude the participants of the preliminary research from the main research. By using quantitative research, items were selected in which at least 23 respondents (57.5\%) expressed high anxiety. Finally, 31 items were selected to constitute the questionnaire from the initial pool of 43 items. Final items included both the information of PEMCS, such as telephone consultation, and public support, such as Kagawa Emergency Support Network. The emergency support network is conducted by 47 municipalities to care for sick children of parents, such as a nuclear family with two-income or single mother [55], and $84.2 \%$ of 310 family support centers conduct the sick child's care as emergency support [56].

The questionnaire also included demographic variables and the situation in which participants used a pediatric emergency institution most recently.

\subsection{Procedures}

This survey was performed as part of a research project entitled "Pediatric Emergency Medical Care in Kagawa Prefecture". Between January and February 2009, the survey was conducted [57]. The questionnaire was handed to parents by a staff member of a kindergarten, nursery school, or health center. The participants were asked to reply only if they consented to participate in the survey. As to the method of returning the questionnaire, mail was used by 36 private kindergartens, 215 nursery schools, and 21 health centers, and the collection method was used at one national and 134 public kindergartens.

In order to avoid duplication in replying to the questionnaire and confirm the number of participants, the survey was conducted as follows: parents with two or more children attending the same kindergarten or nursery school were each handed one questionnaire; the institution maintained a record of the number of questionnaires distributed and the number of remaining questionnaires, and made a report of these figures; and when a guardian had already replied to the questionnaire, she/he was asked to mark in the subsequent questionnaire that she/he had already replied, provide a signature, and return it.

\subsection{Ethical Considerations}

Approval of this study was obtained from the ethical review board of the author's institution (Heisei 20 - 25). This study was administered after obtaining informed consent from the Kagawa Prefectural Government, 17 municipal offices, the principal organization of national and public kindergartens, a private kindergarten federation, and 21 health centers.

\subsection{Data Analyses}

To maintain the quality of the data, all missing data were excluded from the 
candidate data of the scale. All participants were randomly divided into two groups using an odd-even splitting method. First, using one half of the participants, which were assigned an odd number identification (odd number group), exploratory factor analysis (EFA) was performed to generate items and factors for parental anxiety about PEMCS, and the factor structure and reliability of the scale were examined. Second, using the second half of the sample, which was assigned an even number identification (even number group), confirmatory factor analysis (CFA) was performed to test the generality of the extracted factor structure by EFA, and reliability and construct validity for the scale were examined.

All statistical analyses were carried out with SAS 9.2, EFA was conducted by the FACTOR procedure, and CFA was performed by the CALIS procedure.

\subsubsection{Exploratory Factor Analysis and Reliability}

If a high proportion ( $\geq 50 \%$ ) scored the highest (ceiling) or lowest (floor) possible score on an item, this item was excluded as a floor or ceiling effect [58] [59]. EFA was performed using a polychoric correlation due to the categorical nature of the data: four-category Likert scales [60]. Variance inflation factor of more than five suggested that there was multicollinearity between the candidate items [61] [62].

Because unweighted least squares on EFA makes no assumptions of normality [63] and is feasible with large samples [64], unweighted least squares was used for factor extraction as an estimation method, along with a promax rotation. An indicator of the Kaiser-Meyer-Olkin measure of sampling adequacy less than 0.5 suggested that the factor analysis was not appropriate [65] [66] [67]. The decision on how many factors to retain was guided by the use of Cattell's Scree method [65] [66] [68], and the Kaiser rule (factors with an eigenvalue greater than or equal to 1.0 to be retained) [65] [66] [69]. The number of factors that accounted for more than $75 \%$ of the extracted variance was retained [70]. Items were selected for measurement based on the factor pattern matrix using the following criteria: a factor loading above 0.45 on the factor and cross-loadings on other factors of less than 0.32 , which equates to approximately $10 \%$ overlapping variance with the other items in that factor [71]. The item should be removed if the communality is less than 0.5 , because the item does not share much in common with the extracted components, outlier item [66].

The reliability assessments of the scale extracted by EFA were conducted using the polychoric ordinal alpha coefficient, item-rest correlation, and good-poor analysis [72]. Homogeneity was considered acceptable when the polychoric ordinal alpha coefficient was 0.7 or greater [66] [73] [74] [75]. An item-rest correlation of 0.2 was considered a minimum acceptable threshold for retaining items in the scale [76].

\subsubsection{Confirmatory Factor Analysis, Reliability, and Construct Validity}

In order to evaluate fit of the scale structure based on EFA (factorial validity), 
CFA was conducted using the unweighted least squares estimation method and polychoric correlation matrix. The unweighted least squares estimation method for CFA provides more accurate and less variable parameter estimates, more precise standard errors, and better coverage rates [77] [78].

The reliability and validity of the proposed model, and the quality of the individual items were evaluated using component fit results. As the chi-square difference test is highly sensitive to the sample size, model fit was assessed using a combination of fit indices [79]. With regard to the goodness-of-fit index, adjusted goodness-of-fit index, and Bentler-Bonett normed fit index, a value of 0.95 or greater was generally accepted as indicative of an adequate model fit [79] [80]. A root mean square residual or standardized root mean square residual of 0.05 or less was considered a good fit, and that of 0.1 or less was considered an acceptable fit [80].

In order to assess convergent validity, indicator reliability, composite (construct) reliability, and average variance extracted were examined [81]. Indicator reliability was evaluated by the R-squared, which was considered $50 \%$ or more of the variance of the latent factor [82] [83]. Composite reliability estimates the extent to which a set of latent factor indicators share in their measurement of a factor and provides a much less biased estimate of reliability than Cronbach alpha [84] [85]. It was considered adequate if composite reliability was greater than a threshold of 0.7 [81] [83].

Average variance extracted estimates above 0.5 were treated as indications of convergent validity, which means that at least $50 \%$ of the variance in a measurement was due to the hypothesized latent factor [81] [83] [86]. Discriminant validity was evaluated by the Fornell-Larker criterion, in which the average variance extracted estimates of each factor compare to the squared correlations between the respective and other factors (the variance shared between the factors). Discriminant validity was supported if any average variance extracted was greater than any squared correlation between all pairs of factors [83] [86].

\subsubsection{Generalizability}

It is essential for generalizability of scale to demonstrate measurement invariance. Tucker's congruence coefficient, congruence test, and multigroup CFA were conducted to examine measurement invariance of the scale. Tucker's congruence coefficient index assessed the similarity of two factor-loading patterns. Tucker's congruence coefficient exceeding 0.95 suggested that the two factors compared could be considered equal [87] [88]. Congruence test was added as a reliability test, because unlike Tucker's congruence coefficient it does not produce overestimation when a large proportion of highly loading variables are compared. Congruence test was distributed in the range of 0 (similar solutions) until 1 (total discrepancy) [80] [87]. To examine whether the scale held measurement invariance in all demographic variables, multigroup CFA analyses were performed [89] [90]. 


\subsubsection{Usefulness}

Because little is known about the relationship between parental anxiety about PEMCS and the risks of non-urgent PED usage, a suitable scale for verifying concurrent validity of the scale could not be found by literature retrieval. On that account, in order to verify usefulness of the scale, using a Cochran-Mantel-Haenszel test and generalized linear model, the relationship between the scale and vulnerable factors or PEMCS systems was examined.

For example, the relationship between the scale and a certain vulnerable variable, such as single mother, was assessed by means of Cochran-Mantel-Haenszel test, stratified according to other demographic variables. In each vulnerable variable, the common relative risk was calculated in Cochran-Mantel-Haenszel test as the ratio of the proportion of the higher score group ( $\geq$ median) in the other demographic group, versus the higher score group ( $\geq$ median) in the particular demographic group, such as single mother, as a reference group that was inferred with the highest risk of non-urgent usage of PED in a previous study [91] [92] [93]. The Breslow-Day test was used to confirm homogeneity for strata. In addition, generalized linear models which handle non-normal data were employed for the comparison of the scale scores among parental vulnerabilities and PEMCS systems [94] [95].

To clarify the usefulness of the scale on the mechanism clarification for non-urgent usage of pediatric emergency institutions, the relationship among the risk factors of non-urgent usage of the PEMCS, the scale and 8 pediatric emergency institutions that participants used in the immediate past were analyzed. In addition, to analyze certain structural aspects of the scale through the risk factors and non-urgent usage of pediatric emergency institutions, a multiple correspondence analysis was employed as a statistical multivariable analysis that allows the joint study of relational and attributive data [96] [97].

\section{Results}

\subsection{Participants}

Among participants who received a questionnaire, 34,606 questionnaires were evaluated after excluding 5,847 questionnaires (5,256 incomplete questionnaires and 591 duplication questionnaires) from 40,453 distributed questionnaires. Among the 18,043 questionnaires that were returned, 17,452 questionnaires (50.4\%) were evaluated after excluding 591 duplication questionnaires. To constitute the scale, a complete database was constructed from 14,510 (83.1\%) respondents who responded to all 31 items. The demographical variables of the odd number group were not different from those of the even number group (Table $1)$.

\subsection{Data Analysis}

First, EFA was performed to generate items and factors for parental anxiety about PEMCS. 
Table 1. Demographic characteristics of the participants $(\mathrm{N}=14,510)$.

\begin{tabular}{|c|c|c|c|c|}
\hline $\begin{array}{c}\text { Demographic } \\
\text { variable }\end{array}$ & Item & $\begin{array}{c}\text { Odd } \\
\text { number } \\
\text { group } \\
\mathbf{n}=7255 \\
\mathrm{n}\end{array}$ & $\begin{array}{c}\text { Even } \\
\text { number } \\
\text { group } \\
\mathrm{n}=7255 \\
\mathrm{n}\end{array}$ & $P$-value \\
\hline \multirow[t]{4}{*}{ Respondent } & Mother & 6951 & 6951 & $0.994^{\mathrm{a}}$ \\
\hline & Father & 242 & 240 & \\
\hline & Grandparent & 34 & 32 & \\
\hline & Other & 6 & 6 & \\
\hline Age of respondent & $15-19$ & 3 & 1 & $0.776^{\mathrm{b}}$ \\
\hline \multirow[t]{7}{*}{ (Years old) } & $20-24$ & 107 & 130 & \\
\hline & $25-29$ & 916 & 845 & \\
\hline & $30-34$ & 2697 & 2725 & \\
\hline & $35-39$ & 2550 & 2614 & \\
\hline & $40-44$ & 817 & 780 & \\
\hline & $45-49$ & 105 & 103 & \\
\hline & $\geq 50$ & 41 & 39 & \\
\hline \multirow[t]{7}{*}{ Family structure } & Nuclear family, father working & 2373 & 2421 & $0.120^{c}$ \\
\hline & Nuclear family, two incomes & 2817 & 2815 & \\
\hline & $\begin{array}{l}\text { Living together with grandparents, } \\
\text { father working }\end{array}$ & 466 & 474 & \\
\hline & $\begin{array}{l}\text { Living together with } \\
\text { grandparents, two incomes }\end{array}$ & 911 & 852 & \\
\hline & Single mother & 176 & 193 & \\
\hline & $\begin{array}{l}\text { Single father living together } \\
\text { with grandparents }\end{array}$ & 46 & 25 & \\
\hline & $\begin{array}{l}\text { Single mother living together } \\
\text { with grandparents }\end{array}$ & 186 & 171 & \\
\hline \multirow[t]{4}{*}{$\begin{array}{l}\text { Number of } \\
\text { children }\end{array}$} & One & 1485 & 1541 & $0.777^{\mathrm{b}}$ \\
\hline & Two & 3994 & 3904 & \\
\hline & Three & 1515 & 1546 & \\
\hline & Four or more & 226 & 227 & \\
\hline \multirow[t]{6}{*}{ Medical region } & Chusan & 2158 & 2191 & $0.661^{\mathrm{c}}$ \\
\hline & Takamatsu & 3283 & 3204 & \\
\hline & Okawa & 639 & 637 & \\
\hline & Mitoyo & 990 & 1012 & \\
\hline & Shozu & 145 & 162 & \\
\hline & $\begin{array}{l}\text { Naoshima (remote island in the } \\
\text { Takamatsu medical region) }\end{array}$ & 18 & 24 & \\
\hline
\end{tabular}

${ }^{\text {a } F i s h e r ' s ~ e x a c t ~ t e s t . ~}{ }^{b}$ Wilcoxon rank-sum test. ${ }^{c}$ Chi-square test. The total number is different for the missing values. 
Then, CFA was performed to evaluate fit of the scale structure based on EFA.

\subsubsection{Exploratory Factor Analysis and Reliability}

A floor effect or ceiling effect was not observed for all items. As five elements of 465 elements had a variance inflation factor value of more than five, four items were omitted from the polychoric correlation matrix in order to exclude multicollinearity (Appendix 1).

The Kaiser-Meyer-Olkin measure of sampling adequacy was 0.925 , which was a clear indication that it was a high factorability for the sample. On the basis of Cattell's scree test, it was decided to extract four factors, as the decrease of plotted eigenvalues appeared to level off after factor four. Likewise, a four-factor structure was obtained in accordance with the final eigenvalues (11.03, 2.85, $1.69,1.19)$, which were higher than 1.0 , the reference value set by the Kaiser rule. A four-factor solution accounted for $92.29 \%$ of the total variance in the items before rotation.

On the basis of these criteria, a second EFA was conducted, and 15 items out of the original 27 items were retained in the end. Following a promax rotation, loadings of the items on the respective factors all exceeded 0.674 , and there was no item having a cross-loading exceeding 0.195 on the other factors (Table 2). All extracted communalities were greater than the recommended threshold of 0.5. Four factors were labeled as follows: factor one, "anxiety about quality of pediatric emergency medical care services"; factor two, "anxiety about pediatric emergency medical care service system"; factor three, "anxiety about public support"; factor four, "anxiety about private support". The scale was named parental anxiety about pediatric emergency medical care services (PAPEMCS).

The polychoric ordinal alpha coefficient on each factor and scale was above the acceptable threshold of 0.7 , which indicated internal consistency reliability (Table 3). All item-rest of scale correlation coefficients were greater than the minimum acceptable correlation threshold of 0.2. Good-Poor analysis indicated item discrimination.

\subsubsection{Confirmatory Factor Analysis, Reliability, and Construct Validity}

Based on findings from the EFA, CFA was conducted using unweighted least squares as the estimator. To confirm the integrity of the model, the four-factor model was compared with the other two models: the three-factor model which excluded the fourth factor (two items), and the second-order factor model that was selected as the factor model explaining the four first-order factors. Because the combination of fit indices of the four-factor model fulfilled the predetermined level more than those of the other two factor models, the four-factor model was selected as the most acceptable model (Table 4).

For the four-factor model, all the indicator reliabilities, composite reliabilities, and average variance extracted estimates exceeded the recommended thresholds $(0.5,0.7$, and 0.5 , respectively). Hence, the convergent validity was demonstrated. For all four factors, the average variance extracted estimates were greater 
Table 2. Summary of exploratory factor analysis, confirmatory factor analysis, and reliability of PAPEMCS $(\mathrm{N}=14,510)$.

(a)

\begin{tabular}{|c|c|c|c|c|c|c|}
\hline \multirow{2}{*}{$\mathbf{N}$} & \multirow{2}{*}{ Factor (Item) } & \multicolumn{5}{|c|}{ Exploratory factor analysis ${ }^{\mathrm{a}}$} \\
\hline & & F1 & F2 & F3 & F4 & $\mathrm{C}$ \\
\hline & Anxiety about quality of pediatric emergency medical care services (F1) & & & & & \\
\hline 29 & There is no reasonable explanation of the disease and treatment. & 0.956 & -0.059 & -0.032 & 0.040 & 0.87 \\
\hline 31 & No indication regarding the patient's next consultation is given. & 0.898 & -0.064 & 0.032 & 0.010 & 0.76 \\
\hline 26 & Pain and symptoms are not relieved appropriately. & 0.875 & 0.061 & -0.032 & 0.008 & 0.83 \\
\hline 28 & $\begin{array}{l}\text { There is no support for a parent's insufficient experience/knowledge, } \\
\text { anxiety, and concerns. }\end{array}$ & 0.851 & 0.001 & 0.051 & 0.039 & 0.78 \\
\hline 27 & In the emergency consultation hospital, there is no facility to stay at. & 0.760 & 0.195 & -0.033 & -0.040 & 0.75 \\
\hline \multirow[t]{2}{*}{23} & Examinations including X-ray, CT, or blood or urine tests are not available. & 0.683 & 0.187 & 0.058 & -0.067 & 0.64 \\
\hline & Anxiety about pediatric emergency medical care service system (F2) & & & & & \\
\hline 17 & Emergency facilities available change day by day. & -0.023 & 0.860 & 0.099 & -0.086 & 0.73 \\
\hline 18 & The locations of emergency facilities available are unclear. & 0.045 & 0.825 & -0.012 & 0.058 & 0.77 \\
\hline 19 & $\begin{array}{l}\text { It takes over } 30 \text { minutes from home to get to an emergency facility } \\
\text { providing consultation. }\end{array}$ & 0.168 & 0.689 & -0.037 & 0.037 & 0.65 \\
\hline \multirow[t]{2}{*}{16} & There is no facility providing consultation at any time. & 0.124 & 0.674 & -0.020 & 0.105 & 0.64 \\
\hline & Anxiety about public support (F3) & & & & & \\
\hline 3 & Use telephone consultation. & -0.014 & -0.041 & 0.940 & 0.013 & 0.86 \\
\hline 5 & Use Kagawa Emergency Support Network. & 0.016 & 0.006 & 0.731 & 0.014 & 0.55 \\
\hline \multirow[t]{2}{*}{2} & Judge the disease state and take action via the Internet or by cellular phone. & 0.025 & 0.077 & 0.727 & -0.012 & 0.58 \\
\hline & Anxiety about private support (F4) & & & & & \\
\hline 9 & $\begin{array}{l}\text { There is no family member or friend with whom you can } \\
\text { consult about your child's diseases and injuries. }\end{array}$ & 0.020 & -0.004 & 0.028 & 0.953 & 0.93 \\
\hline 10 & There is no family member or friend whom you can ask to take care of your child. & -0.005 & 0.050 & -0.014 & 0.850 & 0.75 \\
\hline
\end{tabular}

(b)

\begin{tabular}{|c|c|c|c|c|c|c|c|c|c|c|c|c|c|}
\hline \multirow{3}{*}{$\mathbf{N}$} & \multirow{3}{*}{$\operatorname{IRC}^{\mathrm{a}, \mathrm{c}}$} & \multicolumn{4}{|c|}{ Good-poor analysis ${ }^{\mathrm{a}, \mathrm{d}}$} & \multicolumn{3}{|c|}{ Confirmatory factor analysis ${ }^{\mathrm{b}}$} & \multirow{2}{*}{\multicolumn{2}{|c|}{$\begin{array}{l}\text { Odd number } \\
\text { group }\end{array}$}} & \multirow{2}{*}{\multicolumn{2}{|c|}{$\begin{array}{l}\text { Even number } \\
\text { group }\end{array}$}} & \multirow{3}{*}{$P$-value } \\
\hline & & \multicolumn{2}{|c|}{$<25 \%$} & \multicolumn{2}{|c|}{$>75 \%$} & \multirow{2}{*}{ SL } & \multirow{2}{*}{ RS } & \multirow{2}{*}{$\begin{array}{c}\text { Error } \\
\text { variance }\end{array}$} & & & & & \\
\hline & & Mean & SD & Mean & SD & & & & Mean & SD & Mean & SD & \\
\hline \multicolumn{14}{|l|}{$\mathrm{F} 1$} \\
\hline 29 & 0.70 & 1.67 & 0.73 & 3.77 & 0.50 & 0.895 & 0.801 & 0.199 & 2.86 & 1.04 & 2.84 & 1.04 & 0.286 \\
\hline 31 & 0.67 & 1.50 & 0.63 & 3.52 & 0.69 & 0.849 & 0.721 & 0.279 & 2.58 & 1.02 & 2.55 & 1.03 & 0.124 \\
\hline 26 & 0.71 & 1.62 & 0.72 & 3.80 & 0.45 & 0.902 & 0.814 & 0.186 & 2.85 & 1.05 & 2.84 & 1.06 & 0.392 \\
\hline 28 & 0.71 & 1.68 & 0.64 & 3.69 & 0.54 & 0.892 & 0.795 & 0.205 & 2.75 & 0.98 & 2.74 & 0.98 & 0.356 \\
\hline 27 & 0.70 & 1.48 & 0.62 & 3.67 & 0.58 & 0.872 & 0.761 & 0.239 & 2.68 & 1.06 & 2.65 & 1.06 & 0.132 \\
\hline 23 & 0.65 & 1.66 & 0.72 & 3.60 & 0.62 & 0.819 & 0.671 & 0.329 & 2.74 & 1.00 & 2.71 & 1.00 & 0.092 \\
\hline \multicolumn{14}{|l|}{$\mathrm{F} 2$} \\
\hline 17 & 0.59 & 1.66 & 0.71 & 3.42 & 0.75 & 0.778 & 0.605 & 0.395 & 2.55 & 0.99 & 2.53 & 0.99 & 0.145 \\
\hline 18 & 0.66 & 1.59 & 0.71 & 3.65 & 0.62 & 0.861 & 0.741 & 0.259 & 2.65 & 1.06 & 2.64 & 1.07 & 0.336 \\
\hline 19 & 0.64 & 1.54 & 0.73 & 3.60 & 0.66 & 0.848 & 0.719 & 0.281 & 2.59 & 1.07 & 2.59 & 1.08 & 0.941 \\
\hline 16 & 0.63 & 1.65 & 0.81 & 3.74 & 0.55 & 0.838 & 0.703 & 0.297 & 2.75 & 1.10 & 2.73 & 1.10 & 0.280 \\
\hline
\end{tabular}


(c)

\begin{tabular}{|c|c|c|c|c|c|c|c|c|c|c|c|c|c|}
\hline \multicolumn{14}{|l|}{ F3 } \\
\hline 3 & 0.33 & 1.57 & 0.73 & 2.54 & 0.95 & 0.834 & 0.696 & 0.304 & 2.06 & 0.89 & 2.05 & 0.89 & 0.715 \\
\hline 5 & 0.31 & 1.71 & 0.77 & 2.63 & 0.93 & 0.747 & 0.558 & 0.442 & 2.18 & 0.89 & 2.17 & 0.89 & 0.431 \\
\hline 2 & 0.36 & 1.71 & 0.77 & 2.78 & 0.92 & 0.826 & 0.683 & 0.317 & 2.26 & 0.92 & 2.24 & 0.93 & 0.190 \\
\hline \multicolumn{14}{|l|}{ F4 } \\
\hline 9 & 0.47 & 1.22 & 0.48 & 2.84 & 1.12 & 0.935 & 0.875 & 0.125 & 1.91 & 1.05 & 1.89 & 1.03 & 0.579 \\
\hline 10 & 0.44 & 1.38 & 0.70 & 3.04 & 1.08 & 0.890 & 0.792 & 0.208 & 2.12 & 1.13 & 2.11 & 1.13 & 0.742 \\
\hline
\end{tabular}

PAPEMCS: Parental anxiety about pediatric emergency medical care services, N: Number, C: Communality, IRC: Item-rest correlation, SL: Standardized loading, RS: R-squared. SD: Standard deviation. ${ }^{a}$ Odd number group $n=7255$ Exploratory factor analysis was conducted using unweighted least squares

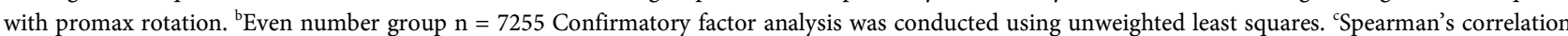
coefficient; all of the probability values showed $P<0.001$. ${ }^{\mathrm{d}} \mathrm{W}$ ilcoxon rank-sum test; all of the probability values showed $P<0.001$. ${ }^{\mathrm{e}} \mathrm{Wilcoxon}$ rank-sum test.

Table 3. Summary of reliability, validity, and generalizability of PAPEMCS $(\mathrm{N}=14,510)$.

(a)

\begin{tabular}{|c|c|c|c|c|c|c|c|c|c|c|c|c|c|}
\hline \multirow{3}{*}{ Factor } & \multicolumn{4}{|c|}{ Correlation matrix ${ }^{\mathrm{a}, \mathrm{d}}$} & \multirow{3}{*}{$\begin{array}{c}\text { Polychoric } \\
\text { ordinal alpha } \\
\text { coefficient }^{\mathrm{a}}\end{array}$} & \multicolumn{4}{|c|}{ Good-poor analysis ${ }^{\mathrm{a}, \mathrm{e}}$} & \multicolumn{4}{|c|}{ Fornell-Larcker criterion ${ }^{\mathrm{b}, \mathrm{f}}$} \\
\hline & \multirow{2}{*}{ F1 } & \multirow{2}{*}{ F2 } & \multirow{2}{*}{ F3 } & \multirow{2}{*}{ F4 } & & \multicolumn{2}{|c|}{$<25 \%$} & \multicolumn{2}{|c|}{$>75 \%$} & \multirow{2}{*}{ F1 } & \multirow{2}{*}{ F2 } & \multirow{2}{*}{ F3 } & \multirow{2}{*}{ F4 } \\
\hline & & & & & & Mean & SD & Mean & SD & & & & \\
\hline $\mathrm{F} 1$ & & & & & 0.949 & 1.60 & 0.49 & 3.67 & 0.34 & 0.761 & & & \\
\hline $\mathrm{F} 2$ & 0.60 & & & & 0.896 & 1.61 & 0.52 & 3.60 & 0.43 & 0.404 & 0.692 & & \\
\hline F3 & 0.24 & 0.32 & & & 0.847 & 1.66 & 0.61 & 2.65 & 0.77 & 0.062 & 0.106 & 0.645 & \\
\hline $\mathrm{F} 4$ & 0.36 & 0.40 & 0.19 & & 0.912 & 1.30 & 0.51 & 2.94 & 1.01 & 0.133 & 0.170 & 0.037 & 0.833 \\
\hline Scale & 0.87 & 0.83 & 0.48 & 0.58 & 0.922 & 1.58 & 0.29 & 3.35 & 0.24 & & & & \\
\hline
\end{tabular}

(b)

\begin{tabular}{|c|c|c|c|c|c|c|c|c|}
\hline \multirow[t]{2}{*}{ Factor } & \multirow{2}{*}{$\begin{array}{l}\text { Composite } \\
\text { reliability }\end{array}$} & \multirow{2}{*}{$\begin{array}{l}\text { Tucker's } \\
\text { congruence } \\
\text { coefficient }^{c}\end{array}$} & \multirow{2}{*}{$\begin{array}{c}\text { Congruence } \\
\text { test }^{c}\end{array}$} & \multicolumn{2}{|c|}{$\begin{array}{l}\text { Odd number } \\
\text { group }^{\mathrm{g}}\end{array}$} & \multicolumn{2}{|c|}{$\begin{array}{l}\text { Even number } \\
\text { group }^{\mathrm{g}}\end{array}$} & \multirow[t]{2}{*}{$P$-value } \\
\hline & & & & Mean & SD & Mean & SD & \\
\hline $\mathrm{F} 1$ & 0.950 & 0.9996 & 0.015 & 2.74 & 0.87 & 2.72 & 0.88 & 0.143 \\
\hline F2 & 0.900 & 0.9995 & 0.014 & 2.64 & 0.88 & 2.62 & 0.89 & 0.317 \\
\hline F3 & 0.845 & 0.9996 & 0.010 & 2.17 & 0.75 & 2.15 & 0.75 & 0.353 \\
\hline F4 & 0.909 & 0.9990 & 0.015 & 2.01 & 1.01 & 2.00 & 1.01 & 0.682 \\
\hline Scale & 0.976 & & & 2.50 & 0.66 & 2.49 & 0.67 & 0.139 \\
\hline
\end{tabular}

PAPEMCS: Parental anxiety about pediatric emergency medical care services. F1: Anxiety about quality of pediatric emergency medical care services, F2: Anxiety about pediatric emergency medical care service system, F3: Anxiety about public support, F4: Anxiety about private support, SD: Standard deviation. ${ }^{a}$ Odd number group, ${ }^{b}$ Even number group, ${ }^{\mathrm{c}}$ Odd number group and even number group. ${ }^{\mathrm{d}}$ Spearman's correlation coefficient; all of the probability values showed $P<0.001$. 'Wilcoxon rank-sum test; all of the probability values showed $P<0.001$. 'The squared correlations between the factors (Spearman's correlation coefficient). Average variant extracted estimates are presented in bold-faced italic type diagonally. ${ }^{\mathrm{g}}$ The mean factor score significantly differed

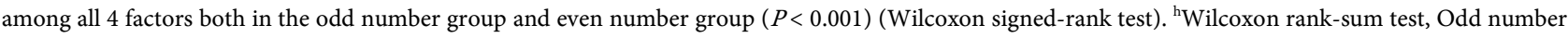
group vs Even number group.

than the squared correlation between all pairs of factors. The Fornell-Lacker criterion of discriminant validity was fulfilled for all factors in the model. 
Table 4. Fit indices for confirmatory factor analysis and fit indices for strict factor invariance.

\begin{tabular}{ccccccc}
\hline Model & Number & GFI & AGFI & NFI & RMSR & SRMSR \\
\hline \multicolumn{2}{c}{ Factor model (Even } & number group) & & & \\
Four-factor model (PAPEMCS) & 7255 & 0.996 & 0.994 & 0.995 & 0.033 & 0.033 \\
Three-factor model & 7255 & 0.996 & 0.994 & 0.995 & 0.035 & 0.035 \\
Second-order factor model & 7255 & 0.996 & 0.994 & 0.995 & 0.034 & 0.034 \\
Multigroup CFA for PAPEMCS: & Strict factorial invariance & & \\
Odd number group, Even number group & 14510 & 0.994 & 0.994 & 0.984 & 0.059 & 0.078 \\
Number of children & 14468 & 0.993 & 0.993 & 0.980 & 0.065 & 0.084 \\
Family structure & 14468 & 0.993 & 0.993 & 0.978 & 0.067 & 0.086 \\
Medical region & 14468 & 0.991 & 0.989 & 0.971 & 0.072 & 0.071 \\
Demographic variables & 14468 & 0.990 & 0.990 & 0.971 & 0.076 & 0.094 \\
\hline
\end{tabular}

PAPEMCS: Parental anxiety about pediatric emergency medical care services. GFI: Goodness of fit index; AGFI: Adjusted goodness of fit index; NFI: Bentler-Bonett normed fit index; RMSR: Root mean square residual; SRMSR: Standardized root mean square residual. Demographic variables included number of children, family structure, and medical region.

\subsubsection{Generalizability}

Between pairs of parallel factors corresponding to EFA of the odd number group and EFA of the even number group, all the Tucker's congruence coefficient indices were 0.999 or greater, and all the congruence tests were 0.015 or less. The results confirmed that both halves were almost identical.

In order to test measurement invariance, multi-group CFA were performed. The level of measurement invariance was assessed through model fit of a hierarchical set of nested multiple group models: configural invariance, metric invariance, scalar invariance and strict factorial invariance. After excluding Naoshima from a multigroup CFA due to small sample size, all the fit indices for strict factor invariance across each of the demographic variables, and all demographic variables, which included number of children, family structure, and medical region, fulfilled the predetermined levels of acceptability.

In summary, the generalizability of the PAPEMCS was demonstrated by the Tucker's congruence coefficients, congruence tests, and strict factorial invariance.

\subsubsection{Usefulness}

Because the generalizability of the PAPEMCS was demonstrated, its usefulness was examined by common relative risk, which was calculated in Cochran-Mantel-Haenszel test as the ratio of the proportion of the higher score group in the other demographic group, versus single mother, parents with only one child, or parents in the Shozu region as reference group, which was inferred with the highest risk of non-urgent usage of PED.

After adjustment for the other demographic variables, a single mother had significantly higher risks of "anxiety about public support" or "anxiety about private support" versus a family living together with grandparents (Table 5). 
Table 5. Common relative risk for PAPEMCS $(\mathrm{N}=14,468)$.

(a)

\begin{tabular}{|c|c|c|c|c|c|c|c|c|c|c|c|c|c|c|}
\hline \multirow{2}{*}{$\begin{array}{l}\text { Demographic } \\
\text { variable }\end{array}$} & \multicolumn{7}{|c|}{$\begin{array}{l}\text { Anxiety about quality of pediatric } \\
\text { emergency medical care services }\end{array}$} & \multicolumn{7}{|c|}{$\begin{array}{l}\text { Anxiety about pediatric emergency medical } \\
\text { care service system }\end{array}$} \\
\hline & $\mathbf{n}$ & $\%$ & CRR & $95 \%$ & CI & $P$-value & BDT & $\mathrm{n}$ & $\%$ & CRR & & $95 \%$ CI & $P$-value & BDT \\
\hline \multicolumn{15}{|l|}{ Family structure ${ }^{\mathrm{a}}$} \\
\hline Single mother & 201 & 54.5 & 1.00 & Refe & ence & & & 204 & 55.3 & 1.00 & & Reference & & \\
\hline NFFW & 2604 & 54.3 & 1.03 & 0.9 & 1.1 & 0.538 & 0.280 & 2496 & 52.1 & 0.96 & 0.9 & 1.1 & 0.461 & 0.643 \\
\hline NFDI & 2979 & 52.9 & 0.99 & 0.9 & 1.1 & 0.833 & 0.820 & 2815 & 50.0 & 0.91 & 0.8 & 1.0 & 0.077 & 0.349 \\
\hline LGFW & 492 & 52.3 & 0.96 & 0.8 & 1.1 & 0.480 & 0.166 & 493 & 52.5 & 0.94 & 0.8 & 1.1 & 0.332 & 0.201 \\
\hline LGDI & 844 & 47.9 & 0.92 & 0.8 & 1.0 & 0.129 & 0.846 & 845 & 47.9 & 0.91 & 0.8 & 1.0 & 0.086 & 0.552 \\
\hline SFG & 30 & 42.3 & 0.76 & 0.6 & 1.0 & 0.056 & 0.543 & 32 & 45.1 & 0.84 & 0.6 & 1.1 & 0.195 & 0.875 \\
\hline SMG & 204 & 57.1 & 1.06 & 0.9 & 1.2 & 0.372 & 0.906 & 206 & 57.7 & 1.05 & 0.9 & 1.2 & 0.439 & 0.437 \\
\hline \multicolumn{15}{|l|}{ Number of children ${ }^{\mathrm{b}}$} \\
\hline One & 1725 & 57.0 & 1.00 & & & & & 1636 & 54.1 & 1.00 & & & & \\
\hline Two & 4171 & 52.8 & 0.93 & 0.9 & 1.0 & 0.001 & 0.049 & 4025 & 51.0 & 0.95 & 0.9 & 1.0 & 0.015 & 0.335 \\
\hline Three & 1544 & 50.4 & 0.89 & 0.9 & 0.9 & 0.001 & 0.217 & 1495 & 48.8 & 0.92 & 0.9 & 1.0 & 0.001 & 0.523 \\
\hline Four or more & 212 & 46.8 & 0.84 & 0.8 & 0.9 & 0.001 & 0.002 & 209 & 46.1 & 0.90 & 0.8 & 1.0 & 0.038 & 0.454 \\
\hline \multicolumn{15}{|l|}{ Medical region $^{c}$} \\
\hline Shozu & 133 & 43.3 & 1.00 & & & & & 146 & 47.6 & 1.00 & & & & \\
\hline Okawa & 676 & 53.0 & 1.20 & 1.0 & 1.4 & 0.008 & 0.261 & 792 & 62.1 & 1.32 & 1.2 & 1.5 & 0.001 & 0.295 \\
\hline Takamatsu & 3621 & 55.8 & 1.25 & 1.1 & 1.4 & 0.001 & 0.073 & 3472 & 53.5 & 1.10 & 1.0 & 1.3 & 0.101 & 0.775 \\
\hline Chusan & 2232 & 51.3 & 1.17 & 1.0 & 1.3 & 0.014 & 0.171 & 2013 & 46.3 & 0.97 & 0.9 & 1.1 & 0.632 & 0.454 \\
\hline Mitoyo & 972 & 48.6 & 1.12 & 1.0 & 1.3 & 0.104 & 0.047 & 930 & 46.5 & 0.99 & 0.9 & 1.1 & 0.888 & 0.335 \\
\hline
\end{tabular}

(b)

\begin{tabular}{|c|c|c|c|c|c|c|c|c|c|c|c|c|c|c|}
\hline \multirow{2}{*}{$\begin{array}{c}\text { Demographic } \\
\text { variable }\end{array}$} & \multicolumn{7}{|c|}{ Anxiety about public support } & \multicolumn{7}{|c|}{ Anxiety about private support } \\
\hline & $\mathbf{n}$ & $\%$ & CRR & \multicolumn{2}{|c|}{$95 \% \mathrm{CI}$} & $P$-value & BDT & $\mathbf{n}$ & $\%$ & CRR & \multicolumn{2}{|c|}{$95 \% \mathrm{CI}$} & $P$-value & BDT \\
\hline \multicolumn{15}{|l|}{ Family structure $^{\mathrm{a}}$} \\
\hline Single mother & 170 & 46.1 & 1.00 & \multicolumn{2}{|c|}{ Reference } & & & 204 & 55.3 & 1.00 & \multicolumn{2}{|c|}{ Reference } & & \\
\hline NFFW & 1943 & 40.5 & 0.90 & 0.8 & 1.0 & 0.078 & 0.267 & 2701 & 56.3 & 1.04 & 0.9 & 1.1 & 0.412 & 0.314 \\
\hline NFDI & 2463 & 43.7 & 0.95 & 0.8 & 1.1 & 0.352 & 0.390 & 2928 & 52.0 & 0.97 & 0.9 & 1.1 & 0.513 & 0.109 \\
\hline LGFW & 355 & 37.8 & 0.80 & 0.7 & 0.9 & 0.004 & 0.740 & 401 & 42.7 & 0.77 & 0.7 & 0.9 & 0.001 & 0.214 \\
\hline LGDI & 761 & 43.2 & 0.93 & 0.8 & 1.1 & 0.288 & 0.108 & 752 & 42.7 & 0.78 & 0.7 & 0.9 & 0.001 & 0.307 \\
\hline SFG & 34 & 47.9 & 0.96 & 0.7 & 1.3 & 0.786 & 0.793 & 43 & 60.6 & 1.05 & 0.8 & 1.3 & 0.658 & 0.078 \\
\hline SMG & 181 & 50.7 & 1.05 & 0.9 & 1.2 & 0.558 & 0.019 & 172 & 48.2 & 0.89 & 0.8 & 1.0 & 0.106 & 0.093 \\
\hline \multicolumn{15}{|c|}{ Number of children ${ }^{\mathrm{b}}$} \\
\hline One & 1365 & 45.1 & 1.00 & & & & & 1689 & 55.8 & 1.00 & & & & \\
\hline Two & 3292 & 41.7 & 0.94 & 0.9 & 1.0 & 0.018 & 0.206 & 4022 & 50.9 & 0.90 & 0.9 & 0.9 & 0.001 & 0.011 \\
\hline Three & 1300 & 42.5 & 0.95 & 0.9 & 1.0 & 0.096 & 0.827 & 1545 & 50.5 & 0.91 & 0.9 & 1.0 & 0.001 & 0.036 \\
\hline Four or more & 173 & 38.2 & 0.85 & 0.7 & 1.0 & 0.010 & 0.008 & 225 & 49.7 & 0.89 & 0.8 & 1.0 & 0.016 & 0.430 \\
\hline \multicolumn{15}{|l|}{ Medical region $^{c}$} \\
\hline Shozu & 127 & 41.4 & 1.00 & & & & & 156 & 50.8 & 1.00 & & & & \\
\hline Okawa & 595 & 46.6 & 1.14 & 1.0 & 1.3 & 0.078 & 0.224 & 632 & 49.5 & 0.99 & 0.9 & 1.1 & 0.822 & 0.224 \\
\hline Takamatsu & 2700 & 41.6 & 1.03 & 0.9 & 1.2 & 0.640 & 0.054 & 3475 & 53.6 & 1.00 & 0.9 & 1.1 & 0.969 & 0.338 \\
\hline Chusan & 1839 & 42.3 & 1.05 & 0.9 & 1.2 & 0.532 & 0.120 & 2234 & 51.4 & 1.00 & 0.9 & 1.1 & 0.963 & 0.585 \\
\hline Mitoyo & 861 & 43.0 & 1.06 & 0.9 & 1.2 & 0.417 & 0.002 & 976 & 48.8 & 1.00 & 0.9 & 1.1 & 0.989 & 0.467 \\
\hline
\end{tabular}


(c)

\begin{tabular}{|c|c|c|c|c|}
\hline \multirow{2}{*}{ Demographic variable } & \multirow{2}{*}{$\mathbf{n}$} & \multicolumn{3}{|c|}{ PAPEMCS } \\
\hline & & Mean & SD & $P$-value ${ }^{\mathrm{d}}$ \\
\hline Family structure & & & & $<0.0024$ \\
\hline Single mother & 369 & 2.56 & 0.66 & A \\
\hline NFFW & 4794 & 2.52 & 0.65 & A \\
\hline NFDI & 5632 & 2.49 & 0.67 & A \\
\hline LGFW & 940 & 2.44 & 0.68 & $\mathrm{AB}$ \\
\hline LGDI & 1763 & 2.40 & 0.68 & B \\
\hline SFG & 71 & 2.42 & 0.68 & $\mathrm{AB}$ \\
\hline SMG & 357 & 2.56 & 0.67 & A \\
\hline Number of children & & & & $<0.0083$ \\
\hline One & 3026 & 2.57 & 0.66 & A \\
\hline Two & 7898 & 2.49 & 0.66 & B \\
\hline Three & 3061 & 2.45 & 0.68 & $\mathrm{BC}$ \\
\hline Four or more & 453 & 2.38 & 0.74 & $\mathrm{C}$ \\
\hline Medical region & & & & $<0.005$ \\
\hline Shozu & 307 & 2.36 & 0.70 & B \\
\hline Okawa & 1276 & 2.56 & 0.62 & $\mathrm{~A}$ \\
\hline Takamatsu & 6487 & 2.55 & 0.62 & A \\
\hline Chusan & 4349 & 2.44 & 0.70 & B \\
\hline Mitoyo & 2002 & 2.41 & 0.71 & B \\
\hline
\end{tabular}

PAPEMCS: Parental anxiety about pediatric emergency medical care services; CRR: Common relative risk; CI: Confidence interval, BDT: Breslow-Day test, SD: Standard deviation, NFFW: Nuclear family, father working, NFDI: Nuclear family, double incomes, LGFW: Living together with grandparents, father working, LGDI: Living together with grandparents, double incomes, SFG: Single father living together with grandparents, SMG: Single mother living together with grandparents. ${ }^{a}$ Adjustment for number of children and medical region. ${ }^{b}$ Adjustment for family structure and medical region. ${ }^{c}$ Adjustment for family structure and number of children. ${ }^{\mathrm{d}}$ The p-value of the generalized linear model of each variable showed $P<0.0001$. Different alphabets indicate a significant difference based on the significance level by the Wilcoxon rank sum test adjusted by the Bonferroni method.

After adjustment for the other demo-graphic variables, only one child showed significantly higher risks of the four factors versus three groups of two or more children. In 8 pediatric emergency institutions that 9,445 participants had used in the most recent past, almost all participants used the pediatric emergency institution in the secondary medical region in which they lived (Table 6). However, some participants living in a secondary medical region providing a pediatric emergency night clinic used the pediatric emergency hospital in a neighboring secondary medical region. In three pediatric emergency night clinics about $80 \%$ of users were for non-urgent usage such as a medical examination and internal medicine, and in three pediatric emergency hospitals about $20 \%$ of users were hospitalized.

Because the PAPEMCS, the risk factors, and non-urgent usage of pediatric emergency institutions were different among pediatric emergency institutions, a 
Table 6. Differences in demographic variables, medical examination and treatment, and parental anxiety about pediatric emergency medical care services among emergency institutions used by participants.

(a)

\begin{tabular}{|c|c|c|c|c|c|c|c|c|c|}
\hline \multirow{3}{*}{ Variable (Item) } & \multirow{3}{*}{$\mathrm{n}$} & \multirow{2}{*}{\multicolumn{2}{|c|}{$\begin{array}{l}\text { Okawa medical } \\
\text { region } \\
\text { Okawa night } \\
\text { emergency } \\
\text { children's clinic }\end{array}$}} & \multicolumn{6}{|c|}{ Takamatsu medical region } \\
\hline & & & & \multicolumn{2}{|c|}{$\begin{array}{l}\text { Takamatsu night } \\
\text { emergency } \\
\text { children's clinic }\end{array}$} & \multicolumn{2}{|c|}{$\begin{array}{l}\text { One group of } \\
\text { hospitals on } \\
\text { rotational duty }\end{array}$} & \multicolumn{2}{|c|}{$\begin{array}{c}\text { Kagawa } \\
\text { university } \\
\text { hospital }\end{array}$} \\
\hline & & $\begin{array}{c}\mathbf{n} \\
\text { Mean }\end{array}$ & $\begin{array}{l}\% \\
\mathrm{SD}\end{array}$ & $\begin{array}{c}\mathrm{n} \\
\text { Mean }\end{array}$ & $\begin{array}{c}\% \\
\mathrm{SD}\end{array}$ & $\begin{array}{c}\mathbf{n} \\
\text { Mean }\end{array}$ & $\begin{array}{l}\% \\
\mathrm{SD}\end{array}$ & $\begin{array}{c}\mathrm{n} \\
\text { Mean }\end{array}$ & $\begin{array}{c}\% \\
\text { SD }\end{array}$ \\
\hline \multicolumn{10}{|l|}{ Family structure $^{\mathrm{a}} \mathrm{n}=9090$} \\
\hline NFFW & 2822 & 115 & 23.2 & 538 & 36.8 & 244 & 37.1 & 166 & 33.6 \\
\hline NFDI & 3831 & 222 & 44.8 & 634 & 43.4 & 271 & 41.3 & 209 & 42.3 \\
\hline LGFW & 639 & 49 & 9.9 & 74 & 5.0 & 36 & 5.5 & 33 & 6.7 \\
\hline LGDI & 1245 & 79 & 15.9 & 121 & 8.3 & 67 & 10.2 & 64 & 13.0 \\
\hline Single mother & 272 & 13 & 2.6 & 52 & 3.6 & 22 & 3.4 & 12 & 2.4 \\
\hline SFG & 49 & 3 & 0.6 & 10 & 0.7 & 2 & 0.3 & 0 & 0.0 \\
\hline SMG & 232 & 15 & 3.0 & 32 & 2.2 & 15 & 2.3 & 10 & 2.0 \\
\hline \multicolumn{10}{|l|}{ Number of children ${ }^{\mathrm{b}} \mathrm{n}=9418$} \\
\hline One & 1948 & 90 & 17.6 & 374 & 24.7 & 151 & 22.2 & 124 & 24.4 \\
\hline Two & 5130 & 299 & 58.4 & 822 & 54.3 & 356 & 52.4 & 263 & 51.7 \\
\hline Three & 2025 & 107 & 20.9 & 283 & 18.7 & 152 & 22.4 & 108 & 21.2 \\
\hline \multirow[t]{2}{*}{ Four or more } & 315 & 16 & 3.1 & 36 & 2.4 & 21 & 3.1 & 14 & 2.8 \\
\hline & & \multicolumn{2}{|c|}{$\mathrm{ABCD}$} & \multicolumn{2}{|c|}{$\mathrm{D}$} & \multicolumn{2}{|c|}{$\mathrm{ABCD}$} & \multicolumn{2}{|c|}{$\mathrm{BCD}$} \\
\hline \multicolumn{10}{|l|}{ Medical region ${ }^{\mathrm{a}} \mathrm{n}=9445$} \\
\hline Okawa & 871 & 486 & 94.2 & 11 & 0.7 & 33 & 4.9 & 151 & 29.4 \\
\hline Takamatsu & 4161 & 29 & 5.6 & 1469 & 96.9 & 599 & 88.1 & 353 & 68.7 \\
\hline Chusan & 2880 & 1 & 0.2 & 31 & 2.0 & 39 & 5.7 & 6 & 1.2 \\
\hline Mitoyo & 1409 & 0 & 0.0 & 3 & 0.2 & 8 & 1.2 & 2 & 0.4 \\
\hline Syozu & 124 & 0 & 0.0 & 2 & 0.1 & 1 & 0.2 & 2 & 0.4 \\
\hline \multicolumn{10}{|l|}{ Medical examination and treatment ${ }^{\mathrm{a}} \mathrm{n}=8620$} \\
\hline A medical examination & 1634 & 106 & 22.2 & 297 & 21.5 & 137 & 23.0 & 67 & 13.8 \\
\hline Internal medicine, suppository etc. & 4566 & 314 & 65.7 & 917 & 66.4 & 217 & 36.4 & 216 & 44.4 \\
\hline Infusion, inhalation etc. & 1369 & 58 & 12.1 & 167 & 12.1 & 100 & 16.8 & 103 & 21.2 \\
\hline Hospitatization & 1051 & 0 & 0.0 & 0 & 0.0 & 143 & 24.0 & 100 & 20.6 \\
\hline \multicolumn{10}{|l|}{ PAPEMCS $^{\mathrm{b}}$} \\
\hline \multirow{2}{*}{\multicolumn{2}{|c|}{$\begin{array}{l}\text { Parental anxiety about quality of pediatric emergency } \\
\text { medical care services }\end{array}$}} & 2.74 & 0.8 & 2.79 & 0.8 & 2.90 & 0.8 & 2.78 & 0.9 \\
\hline & & \multicolumn{2}{|c|}{ B } & \multicolumn{2}{|c|}{ B } & \multicolumn{2}{|c|}{ A } & \multicolumn{2}{|c|}{$\mathrm{AB}$} \\
\hline \multirow{2}{*}{\multicolumn{2}{|c|}{$\begin{array}{l}\text { Parental anxiety about pediatric emergency medical } \\
\text { care service system }\end{array}$}} & 2.87 & 0.9 & 2.73 & 0.8 & 2.82 & 0.8 & 2.65 & 0.9 \\
\hline & & \multicolumn{2}{|c|}{$\mathrm{A}$} & \multicolumn{2}{|c|}{$\mathrm{BC}$} & & & & \\
\hline 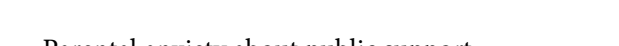 & & 2.23 & 0.7 & 2.12 & 0.7 & 2.18 & 0.7 & 2.23 & 0.8 \\
\hline Parental anxiety about public support & & & & & & & & & \\
\hline Parental anxiety about private support & & 1.92 & 1.0 & 2.08 & 1.0 & 2.07 & 1.0 & 2.10 & 1.1 \\
\hline & & & & & & & & & \\
\hline Scale & & 2.55 & 0.7 & 2.55 & 0.6 & 2.63 & 0.6 & 2.54 & 0.7 \\
\hline scale & & & & & & & & & \\
\hline
\end{tabular}


(b)

\begin{tabular}{|c|c|c|c|c|c|c|c|c|}
\hline \multirow{3}{*}{ Variable (Item) } & \multirow{2}{*}{\multicolumn{2}{|c|}{$\begin{array}{l}\text { Chusan medical } \\
\text { region } \\
\text { Kagawa national } \\
\text { children's hospital }\end{array}$}} & \multirow{2}{*}{\multicolumn{2}{|c|}{$\begin{array}{l}\text { Mitoyo medical } \\
\text { region } \\
\text { Mitoyo night } \\
\text { emergency } \\
\text { children's clinic }\end{array}$}} & \multicolumn{4}{|c|}{ Kagawa prefecture } \\
\hline & & & & & \multicolumn{2}{|c|}{ Family doctor } & \multicolumn{2}{|c|}{$\begin{array}{l}\text { Holiday on duty } \\
\text { doctor }\end{array}$} \\
\hline & $\begin{array}{c}\mathrm{n} \\
\text { Mean }\end{array}$ & $\begin{array}{c}\% \\
\text { SD }\end{array}$ & $\begin{array}{c}n \\
\text { Mean }\end{array}$ & $\begin{array}{c}\% \\
\text { SD }\end{array}$ & $\begin{array}{c}\mathbf{n} \\
\text { Mean }\end{array}$ & $\begin{array}{c}\% \\
\text { SD }\end{array}$ & $\begin{array}{c}\mathbf{n} \\
\text { Mean }\end{array}$ & $\begin{array}{c}\% \\
\text { SD }\end{array}$ \\
\hline \multicolumn{9}{|l|}{ Family structure $^{a} n=9090$} \\
\hline NFFW & 688 & 27.0 & 134 & 23.8 & 382 & 30.5 & 555 & 34.4 \\
\hline NFDI & 1062 & 41.6 & 209 & 37.1 & 512 & 40.8 & 712 & 44.2 \\
\hline LGFW & 211 & 8.3 & 57 & 10.1 & 92 & 7.3 & 87 & 5.4 \\
\hline LGDI & 447 & 17.5 & 132 & 23.5 & 166 & 13.2 & 169 & 10.5 \\
\hline Single mother & 68 & 2.7 & 11 & 2.0 & 48 & 3.8 & 46 & 2.9 \\
\hline SFG & 15 & 0.6 & 2 & 0.4 & 14 & 1.1 & 3 & 0.2 \\
\hline SMG & 62 & 2.4 & 18 & 3.2 & 40 & 3.2 & 40 & 2.5 \\
\hline \multicolumn{9}{|l|}{ Number of children $^{\mathrm{b}} \mathrm{n}=9418$} \\
\hline One & 484 & 18.2 & 113 & 19.4 & 252 & 19.3 & 360 & 21.9 \\
\hline Two & 1431 & 53.7 & 316 & 54.1 & 711 & 54.4 & 932 & 56.7 \\
\hline Three & 647 & 24.3 & 135 & 23.1 & 282 & 21.6 & 311 & 18.9 \\
\hline \multirow{2}{*}{ Four or more } & 105 & 3.9 & 20 & 3.4 & 62 & 4.7 & 41 & 2.5 \\
\hline & \multicolumn{2}{|c|}{ A } & \multicolumn{2}{|c|}{$\mathrm{ABC}$} & \multicolumn{2}{|c|}{$\mathrm{AB}$} & \multicolumn{2}{|c|}{$\mathrm{CD}$} \\
\hline \multicolumn{9}{|l|}{ Medical region ${ }^{\mathrm{a}} \mathrm{n}=9445$} \\
\hline Okawa & 9 & 0.3 & 0 & 0.0 & 87 & 6.7 & 94 & 5.7 \\
\hline Takamatsu & 78 & 2.9 & 6 & 1.0 & 502 & 38.4 & 1125 & 68.3 \\
\hline Chusan & 2025 & 75.7 & 6 & 1.0 & 431 & 33.0 & 341 & 20.7 \\
\hline Mitoyo & 562 & 21.0 & 575 & 98.0 & 201 & 15.4 & 58 & 3.5 \\
\hline Syozu & 2 & 0.1 & 0 & 0.0 & 87 & 6.7 & 30 & 1.8 \\
\hline \multicolumn{9}{|l|}{ Medical examination and treatment ${ }^{\mathrm{a}} \mathrm{n}=8620$} \\
\hline A medical examination & 499 & 20.5 & 96 & 19.2 & 154 & 12.4 & 278 & 18.5 \\
\hline Internal medicine, suppository etc. & 1043 & 42.8 & 280 & 55.9 & 604 & 48.8 & 975 & 64.9 \\
\hline Infusion, inhalation etc. & 321 & 13.2 & 125 & 25.0 & 320 & 25.8 & 175 & 11.7 \\
\hline Hospitatization & 573 & 23.5 & 0 & 0.0 & 161 & 13.0 & 74 & 4.9 \\
\hline \multicolumn{9}{|l|}{ PAPEMCS $^{\mathrm{b}}$} \\
\hline Parental anxiety about quality of pediatric & 2.59 & 1.0 & 2.52 & 1.0 & 2.72 & 0.9 & 2.80 & 0.8 \\
\hline emergency medical care services & \multicolumn{2}{|c|}{$\mathrm{C}$} & \multicolumn{2}{|c|}{$\mathrm{C}$} & \multicolumn{2}{|c|}{ B } & \multicolumn{2}{|c|}{$\mathrm{AB}$} \\
\hline \multirow{2}{*}{$\begin{array}{l}\text { Parental anxiety about pediatric emergency } \\
\text { medical care service system }\end{array}$} & 2.38 & 1.0 & 2.40 & 1.0 & 2.64 & 0.9 & 2.69 & 0.8 \\
\hline & \multicolumn{2}{|c|}{$\mathrm{D}$} & \multicolumn{2}{|c|}{$\mathrm{D}$} & \multicolumn{2}{|c|}{$\mathrm{C}$} & \multicolumn{2}{|c|}{$\mathrm{C}$} \\
\hline Parental anxiety about public sunnort & 2.20 & 0.8 & 2.19 & 0.8 & 2.19 & 0.8 & 2.16 & 0.7 \\
\hline & & & & & & & & \\
\hline Parental anxiety about private sunnort & 1.97 & 1.0 & 1.96 & 1.0 & 2.03 & 1.0 & 2.06 & 1.0 \\
\hline & & & & & & & & \\
\hline Scole & 2.37 & 0.7 & 2.34 & 0.7 & 2.50 & 0.7 & 2.54 & 0.6 \\
\hline tart & & & & & & & & \\
\hline
\end{tabular}

N: Number, SD: Standard deviation, NFFW: Nuclear family, father working, NFDI: Nuclear family, double incomes, LGFW: Living together with grandparents, father working, LGDI: Living together with grandparents, double incomes, SFG: Single father living together with grandparents, SMG: Single mother living together with grandparents, PAPEMCS: Parental anxiety about pediatric emergency medical care service. ${ }^{a}$ Chi square test $P<0.0001$, ${ }^{b}$ Steel-Dwass test (Tukey-Kramer test) Difference in alphabet indicates a significant difference among 8 emergency institutions $(P<0.05)$. 
multiple correspondence analysis was employed to examine these relationships more precisely.

All of the higher score groups of the PAPEMCS were close in distance to a demo-graphic cluster including nuclear family, single parent and few children, the treatment for slight disease, and 3 pediatric emergency medical institutions in Takamatsu (Figure 1). All of the lower score groups of the PAPEMCS were close to another demographic cluster including the family living together with grandparents, more children, the treatment for moderate or severe disease, and pediatric emergency medical institutions in three secondary medical regions (Chusan, Mitoyo and Chozu). One cluster was independently constituted from a pediatric emergency night clinic in Okawa secondary medical region (Okawa).

In addition, although generalized linear models and multiple comparison using Wilcoxon rank-sum tests were not adjusted by two other variables, the differences in PAPEMCS scores among parental vulnerabilities and PEMCS systems were similar to the results examined by multiple correspondence analysis.

\section{Discussion}

\subsection{Strengths of the PAPEMCS}

The purpose of the present study was to develop a scale of parental anxiety about PEMCS and provide evidence of initial reliability, validity, generalizability and usefulness for this new scale. The strengths of this study are that the fairly large sample allowed us to develop a psychometrically robust scale, and the participants possessed high representativeness that fulfilled the research requirements: multifarious family structure, various PEMCS systems, and diverse geographical features.

\subsection{Content Validity of Four Factors}

EFA and CFA implemented for odd and even number groups, respectively, provided one general construct and four factors as evidence of the validity of the scale structure.

The first factor, "anxiety about quality of PEMCS", constituted anxiety about two characteristics of pediatric emergency institutions (resources and family-centered care) that are important concerns of parents with sick children. The second factor, "anxiety about PEMCS system", was comprised of anxiety about accessibility to pediatric emergency institutions. The fourth factor, "anxiety about private support", was comprised of anxiety about a shortage of private support. These three factors demonstrate content validity, because they are consistent with the parental anxieties about PED usage in previous studies, or correspond with the reasons of parents visiting a PED for a child with a non-urgent condition [5] [23] [25] [26] [28] [29] [39] [51] [54].

The third factor, "anxiety about public support," was constituted of not only pre-hospital care (telephone consultation, information on website) but also post-hospital care (Kagawa Emergency Support Network). The factor represents 


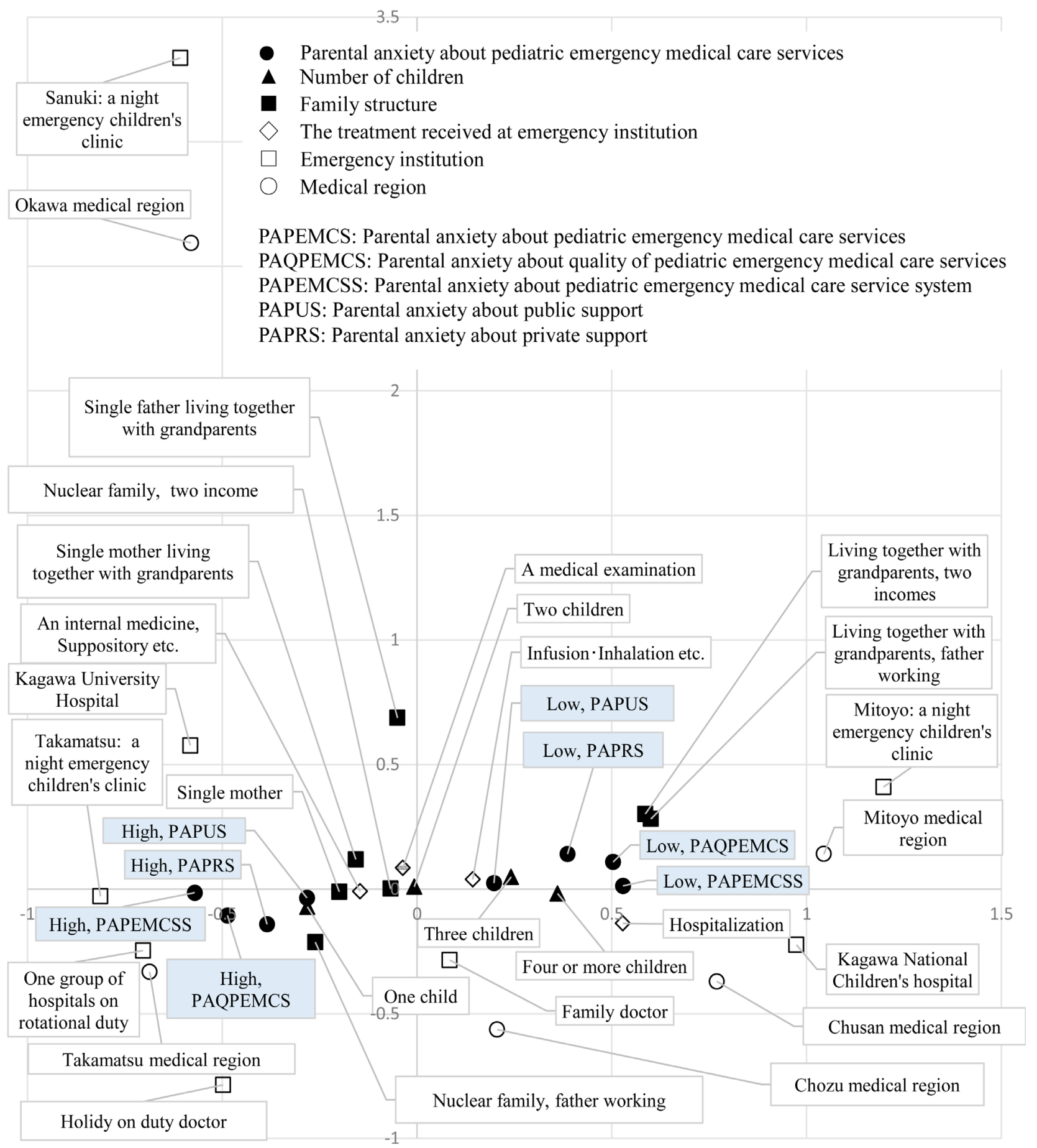

Figure 1. Profiles of PAPEMCS, demographic variables and emergency medical usage in a two-dimensional plot $(\mathrm{N}=8,620)$.

parental anxiety not only about pediatric emergency institutions but also all care services for sick children. In addition, the emergency support network service for the sick child care increased from 47 municipalities in 2009 to 142 municipalities in 2013 [98].

Hence, the PAPEMCS demonstrated content validity in that the four factors were comprehensively constituted from parental anxiety about PEMCS. 


\subsection{Reliability, Validity and Generalizability}

Excellent evidence was demonstrated about the internal homogeneity and reliability of the PAPEMCS. All reliability estimates (the polychoric ordinal alpha coefficients and item-rest correlations), the item discrimination (good-poor analysis), fit indices for CFA (goodness-of-fit index, adjusted goodness-of-fit index, Bentler-Bonett normed fit index, root mean square residual, and standardized root mean square residual), the convergent validity (indicator reliabilities, composite reliabilities, and average variance extracteds), and the discriminant validity (Fornell-Lacker criterion) fulfilled the acceptability thresholds.

The generalizability of the PAPEMCS was revealed from evidence of Tucker's congruence coefficients, congruence tests, and strict factor invariance across 16 demographic groups that showed substantial heterogeneity. Because strict factorial invariance reveals that differences in scale scores reflect true differences on the construct being measured, the present findings provided convincing evidence that the PAPEMCS enables comparing parental anxiety about PEMCS among various contexts, such as parental vulnerabilities and PEMCS systems.

\subsection{Usefulness}

The PAPEMCS offered some useful findings for PEMCS. First of all, parents who are known as non-urgent PED users, single parent and parents with a few number of children, showed higher anxiety of PEMCS. Using multiple correspondence analysis, the study demonstrated the new finding that the mutual close relationship was among higher groups of the PAPEMCS, the risk factors of non-urgent users of PEMCS (single parent, nuclear family and parents with a few number of children), and non-urgent users whose child received a medical examination or an internal medicine at pediatric emergency institution. The other finding was the mutual close relationship among lower groups of the PAPEMCS, family living together with grandparents, parents with three children or more, and users whose child received treatment by infusion or hospitalization at pediatric emergency institutions. These results suggest the possibility that the shortage of private support to parents or the shortage of the parents' child rearing experience, caused higher parental anxiety about PEMCS and resulted in non-urgent usage of pediatric emergency institutions.

Regarding the reason that 3 pediatric medical institutions in Takamatsu were related to the higher group of the PAPEMCS and 2 pediatric medical institutions in Chusan and Mitoyo were related to the lower group of the PAPEMCS; this may be due to the difference in family structures among the 5 medical regions [99]. More nuclear families, who are a risk factor for non-urgent PEMCS use, lived in Takamatsu than in the other 4 medical regions, and families living together with grandparents were fewer in Takamatsu than in the other 4 medical regions: nuclear family/family living together with grandparents, Takamatsu $84 \% / 8 \%$, Okawa $71 \% / 21 \%$, Chusan $76 \% / 14 \%$, Mitoyo $66 \% / 26 \%$, Syozu $74 \% / 16 \%$. These results revealed that family vulnerability influenced the usage tendency for 
the pediatric emergency institution in the residing region.

Also, for parents living in a region providing the pediatric emergency night clinic the anxiety regarding PEMCS or usage of a pediatric emergency institution may be influenced by the pediatric emergency system of a neighboring region. Although the PEMCS system of Mitoyo is the same as that of Okawa, parents in Mitoyo can use a pediatric hospital that is available at the primary level of PEMCS 24 hours a day in Chusan, which is a neighboring region. Due to this, parents in Mitoyo were not very anxious about PEMCS and were at a lower rate of non-urgent users. In Okawa, parents did not use the secondary pediatric emergency hospital rotated every day and instead used Kagawa University hospital (tertiary) in Takamatsu. By using the PAPEMCS, this research suggests, at a minimum, the importance of being able to consult an emergency night hospital for sick children at the secondary level of PEMCS. The results suggest that parental anxieties regarding PEMCS are influenced by their vulnerability, and the convenience of a high quality of PEMCS. The PAPEMCS explained the mechanisms by which multifarious risks cause non-urgent use of pediatric emergency institutions, and which were not explained up to the present.

By expansion of the pediatric medical regions in 2014, the regions were reorganized with 231 regions having a pediatric emergency medical core hospital or a pediatric emergency center, and 69 regions not having a hospital (center) [100]. In recent years, some hospitals have provided an emergency medical system in the same way as emergency departments in the U.S., and, for example, these hospitals include Kagawa National Children's Hospital (The present name: Shikoku Medical Center for Children and Adults), Japanese Red Cross Kumamoto Hospital, and the National Center for Child Health and Development. Considering the developmental situation of the PEMCS system in Japan, the findings of this study have established that the PAPEMCS is useful to comprehensively clarify the mechanisms of PEMCS usage.

\subsection{Limitation and Future Studies}

This study has several limitations regarding the development of the PAPEMCS. First, the findings of this study were limited to the participants and PEMCS systems that we investigated. For example, this study were not able to examine if the U. S. type of pediatric emergency medical system helps vulnerable family to decrease their anxiety of PEMCS or their non-urgent usage of PEMCS. Second, most participants were limited to parents with a child who goes to a kindergarten or day-care center. There is a possibility that it is inappropriate to generalize the findings to parents with an infant. Third, the participants were parents in only one prefecture, which has a temperate climate and is a small region. In addition, the results could not reveal parental anxiety about PEMCS for those residing in Naoshima (only one clinic) due to the small sample size. For confirming the generalizability of the PAPEMCS, it is important to examine parents with an infant and parents living under every geographical condition: big cities, de- 
populated areas, and doctor-less villages.

Lastly, the study did not examine the effects of public support, such as a telephone triage against non-urgent usage in PEMCS. It is possible to examine such effects in more detail by analyzing the PAPEMCS, in addition to intervention variables, risk factors such as a single parent, and non-urgent PEMCS usage.

\section{Conclusion}

The fact that all of the calculated measures for assessing reliability, validity and generalizability reached the recommended limit values demonstrated the reliability, validity and generalizability of the PAPEMCS. The fact that there was a close relationship among the PAPEMCS, individual factors and the PEMCS system has established the usefulness of the PAPEMCS. The PAPEMCS is useful to quantify the contents and extent of parental anxiety about PEMCS, and to clarify the mechanisms of non-urgent PEMCS usage.

\section{Acknowledgements}

We are very grateful to all those who participated in this study. This study was funded by Grants-in-Aid for scientific research expenses of the Japan Society for the Promotion of Science (20659352).

\section{Conflict of Interest Disclosure}

The authors have no conflicting interest in this study.

\section{References}

[1] American Academy of Pediatrics Committee on Pediatric Emergency Medicine (2004) Over Crowding Crisis in Our Nation's Emergency Departments: Is Our Safety Net Unraveling? Pediatrics, 114, 878-888.

http://pediatrics.aappublications.org/content/pediatrics/114/3/878.full.pdf https://doi.org/10.1542/peds.2004-1287

[2] Moskop, J.C., Sklar, D.P., Geiderman, J.M., Schears, R.M. and Bookman, K.J. (2009) Emergency Department Crowding, Part 1-Concept, Causes, and Moral Consequences. Annals of Emergency Medicine, 53, 605-611. https://doi.org/10.1016/j.annemergmed.2008.09.019

[3] Richardson, L.D. and Hwang, U. (2001) Access to Care: A Review of the Emergency Medicine Literature. Academic Emergency Medicine, 8, 1030-1036. https://doi.org/10.1111/j.1553-2712.2001.tb01111.x

[4] Massin, M. and Lepage, P. (2002) Observations and Reflections on the Management of Pediatric Emergencies in Belgium. Revue Medicale De Liege, 57, 591-598.

[5] Berry, A., Brousseau, D., Brotanek, J.M., Tomany-Korman, S. and Flores, G. (2008) Why Do Parents Bring Children to the Emergency Department for Nonurgent Conditions? A Qualitative Study. Ambulatory Pediatrics, 8, 360-367. http://www.academicpedsjnl.net/article/S1530-1567(08)00161-5/pdf

[6] Ganapathy, S., Lim, S.Y., Kua, J.P. and Ng, K.C. (2015) Non-Urgent Paediatric Emergency Department Visits: Why Are They So Common? A Singapore Perspective. ANNALS Academy of Medicine Singapore, 44, 269-271. 
[7] Brousseau, D.C., Hoffmann, R.G., Nattinger, A.B., Flores, G., Zhang, Y. and Gorelick, M. (2007) Quality of Primary Care and Subsequent Pediatric Emergency Department Utilization. Pediatrics, 119, 1131-1138.

http://pediatrics.aappublications.org/content/pediatrics/119/6/1131.full.pdf https://doi.org/10.1542/peds.2006-3518

[8] Ohns, M.J., Oliver-McNeil, S., Nantais-Smith, L.M. and George, N.M. (2016) Nonurgent Use of the Emergency Department by Pediatric Patients: A Theory-Guided Approach for Primary and Acute Care Pediatric Nurse Practitioners. Journal of Pediatric Health Care, 30, 339-346. https://doi.org/10.1016/j.pedhc.2015.09.003

[9] Goh, G.L., Huang, P., Kong, M.C., Chew, S.P. and Ganapathy, S. (2016) Unplanned Reattendances at the Paediatric Emergency Department within 72 Hours: A One-Year Experience in KKH. Singapore Medical Journal, 57, 307-313. https://doi.org/10.11622/smedj.2016105

[10] Brousseau, D.C., Bergholte, J. and Gorelick, M.H. (2004) The Effect of Prior Interactions with a Primary Care Provider on Nonurgent Pediatric Emergency Department Use. Archives of Pediatrics and Adolescent Medicine, 158, 78-82. http://jamanetwork.com/journals/jamapediatrics/fullarticle/485603

[11] Kennedy, T.J., Purcell, L.K., LeBlanc, J.C. and Jangaard, K.A. (2004) Emergency Department Use by Infants Less than 14 Days of Age. Pediatric Emergency Care, 20, 437-442. https://doi.org/10.1097/01.pec.0000132216.65600.1b

[12] Phelps, K., Taylor, C., Kimmel, S., Nagel, R., Klein, W. and Puczynski, S. (2000) Factors Associated with Emergency Department Utilization for Nonurgent Pediatric Problems. Archives of Family Medicine, 9, 1086-1092.

https://triggered.clockss.org/ServeContent?rft_id=info:doi/10.1001/archfami.9.10.10 $\underline{86}$

[13] Wier, L.M., Yu, H., Owens, P.L. and Washington, R. (2013) Overview of Childrenthe Emergency Department, 2010: Statistical Brief \#157. Agency for Healthcare Research and Quality, 1-12. http://www.hcup-us.ahrq.gov/reports/statbriefs/sb157.pdf

[14] Chin, N.P., Goepp, J.G., Malia, T., Harris, L. and Poordabbagh, A. (2006) Nonurgent Use of a Pediatric Emergency Department: A Preliminary Qualitative Study. Pediatric Emergency Care, 22, 22-27. https://doi.org/10.1097/01.pec.0000195756.74328.21

[15] Drummond, J., Schnirer, L., So, S., Mayan, M., Williamson, D.L., Bisanz, J., et al. (2014) The Protocol for the Families First Edmonton Trial (FFE): A Randomized Community-Based Trial to Compare Four Service Integration Approaches for Families with Low-Income. BMC Health Services Research, 14, 1-12. https://www.ncbi.nlm.nih.gov/pmc/articles/PMC4060625/pdf/1472-6963-14-223.pdf https://doi.org/10.1186/1472-6963-14-223

[16] Matziou, V., Brokalaki, H., Kyritsi, H., Perdikaris, P., Gymnopoulou, E. and Merkouris, A. (2008) What Greek Mothers Know about Evaluation and Treatment of Fever in Children: An Interview Study. International Journal of Nursing Studies, 45, 829-836.

http://ac.els-cdn.com/S0020748907001046/1-s2.0-S0020748907001046-main.pdf?_ti $\mathrm{d}=47 \mathrm{f} 47 \mathrm{dca}-7063-11 \mathrm{e} 7-\mathrm{a} 206-00000 \mathrm{aacb35e} \& \mathrm{acdnat}=1500895917 \_\mathrm{a} 9 \mathrm{~d} 39 \mathrm{~b} 2 \mathrm{f} 9 \mathrm{a} 769 \mathrm{f9f}$ c9db0cc160ee9036 https://doi.org/10.1016/j.ijnurstu.2006.04.021

[17] Morrison, A.K., Chanmugathas, R., Schapira, M.M., Gorelick, M.H., Hoffmann, R.G. and Brousseau, D.C. (2014) Caregiver Low Health Literacy and Nonurgent Use of the Pediatric Emergency Department for Febrile Illness. Academic Pediatrics, 14, 
505-509.

https://www.ncbi.nlm.nih.gov/pmc/articles/PMC4149856/pdf/nihms598945.pdf https://doi.org/10.1016/j.acap.2014.05.001

[18] Morrison, A.K., Schapira, M.M., Gorelick, M.H., Hoffmann, R.G. and Brousseau, D.C. (2014) Low Caregiver Health Literacy Is Associated with Higher Pediatric Emergency Department Use and Nonurgent Visits. Academic Pediatrics, 14, 309-314.

https://www.ncbi.nlm.nih.gov/pmc/articles/PMC4003496/pdf/nihms556915.pdf https://doi.org/10.1016/j.acap.2014.01.004

[19] Salami, O., Salvador, J. and Vega, R. (2012) Reasons for Nonurgent Pediatric Emergency Department Visits: Perceptions of Health Care Providers and Caregivers. Pediatric Emergency Care, 28, 43-46. https://doi.org/10.1097/PEC.0b013e31823f2412

[20] Neill, S.J., Jones, C.H., Lakhanpaul, M., Roland, D.T. and Thompson, M.J. (2016) Parents' Help-Seeking Behaviours during Acute Childhood Illness at Home: A Contribution to Explanatory Theory. Journal of Child Health Care, 20, 77-86. https://doi.org/10.1177/1367493514551309

[21] Neill, S.J. (2008) Family Management of Acute Childhood Illness at Home: A Grounded Theory Study. PhD Thesis, Nightingale School of Nursing and Midwifery, King's College London, London, UK.

[22] Parkinson, G.W., Gordon, K.E., Camfield, C.S. and Fitzpatrick, E.A. (1999) Anxiety in Parents of Young Febrile Children in a Pediatric Emergency Department: Why Is It Elevated? Clinical Pediatrics, 38, 219-226. https://doi.org/10.1177/000992289903800403

[23] Kubicek, K., Liu, D., Beaudin, C., Supan, J., Weiss, G., Lu, Y., et al. (2012) A Profile of Nonurgent Emergency Department Use in an Urban Pediatric Hospital. Pediatric Emergency Care, 28, 977-984.

https://www.ncbi.nlm.nih.gov/pmc/articles/PMC3464348/pdf/nihms352822.pdf https://doi.org/10.1097/PEC.0b013e31826c9aab

[24] Richier, P., Gocko, X., Mory, O., Trombert-Paviot, B. and Patural, H. (2015) Étude épidémiologique des consultations précoces de nouveau-nés aux services d'accueil des urgences pédiatriques. [An Epidemiological Study of Early Consultations of Newborns in Pediatric Emergency Units.] Archives de Pédiatrie, 22, 135-140. https://doi.org/10.1016/j.arcped.2014.10.004

[25] Rousseau, D.C., Nimmer, M.R., Yunk, N.L., Nattinger, A.B. and Greer, A. (2011) Noncurrent Emergency-Department Care: Analysis of Parent and Primary Physician Perspectives. Pediatrics, 127, e375-e381.

http://pediatrics.aappublications.org/content/pediatrics/127/2/e375.full.pdf https://doi.org/10.1542/peds.2010-1723

[26] Graham, J.M., Fitzpatrick, E.A. and Black, K.J. (2010) “My Child Can’t Keep Anything Down!" Interviewing Parents Who Bring Their Preschoolers to the Emergency Department for Diarrhea, Vomiting, and Dehydration. Pediatric Emergency Care, 26, 251-256. https://doi.org/10.1097/PEC.0b013e3181d6d9de

[27] Hugenholtz, M., Bröer, C. and van Daalen, R. (2009) Apprehensive Parents: A Qualitative Study of Parents Seeking Immediate Primary Care for Their Children. British Journal of General Practice, 59, 173-179. https://www.ncbi.nlm.nih.gov/pmc/articles/PMC2648915/pdf/bjgp59-173.pdf https://doi.org/10.3399/bjgp09X394996

[28] Watanabe, S., Nakazawa, M., Eto, Y., Ichikawa, K., Mori T., Tanaka, A., et al. (2006) Family Needs in Pediatric Emergency Department Use in Japan. The Journal of the Japan Pediatric Society, 110, 696-702. 
[29] Fieldston, E.S., Alpern, E.R., Nadel, F.M., Shea, J.A. and Alessandrini, E.A. (2012) A Qualitative Assessment of Reasons for Nonurgent Visits to the Emergency Department: Parent and Health Professional Opinions. Pediatric Emergency Care, 28, 220-225. https://doi.org/10.1097/PEC.0b013e318248b431

[30] O’Malley, P.J., Brown, K. and Krug, S.E. and Committee on Pediatric Emergency Medicine (2008) Patient- and Family-Centered Care of Children in the Emergency Department. Pediatrics, 122, e511-e521.

http://pediatrics.aappublications.org/content/pediatrics/122/2/e511.full.pdf

[31] Health Policy Unit, Graduate School of Public Policy, The University of Tokyo (2014) RH-PAC Community Medical Vision/The Guideline of Regional Medical Treatment Plan: Pediatric Medicine. [Cited 25 July 2017.] Available from URL: http://www.pp.u-tokyo.ac.jp/HPU/seminar/2014-10-12/d/Guideline_F21.pdf

[32] Japan Pediatric Society (2010) Reformation of Pediatric Emergency Medical Service Advanced by Japan Pediatric Society. [Cited 25 July 2017.] Available from URL: http://jpsmodel.umin.jp/vision.html

[33] Ehara, A. (2009) Are City Population and the Number of Emergency Medical Facilities Correlated? Pediatrics International, 51, 258-259.

http://onlinelibrary.wiley.com/doi/10.1111/j.1442-200X.2008.02694.x/abstract

[34] Guidance of Medical Service Division, Health Policy Bureau, Ministry of Health, Labour and Welfare (2008) Maintenance of the Pediatric Emergency Medical Care Service System.

http://www.wam.go.jp/wamappl/bb13GS40.nsf/0/1a5b9ff439e1d68a49257405001ec d3c/\$FILE/20080307_5shiryou5_1.pdf

[35] Ministry of Health, Labour and Welfare (2009) Reference Materials, Pediatric Emergency Medical Care. http://www.mhlw.go.jp/shingi/2009/10/dl/s1030-17c.pdf

[36] Mashiko, K. (2009) Function and Role of Pre-Hospital Transportation in Tertiary Emergency Medical Care. IA TSS Review, 34, 286-292.

http://www.iatss.or.jp/common/pdf/publication/iatss-review/34-3-04.pdf

[37] Yamamoto, Y and Sakamoto, T. (2012) The Health and Labour Science Research, Study on Promotion of the Emergency Care System, the Present Situation of Emergency and Critical Care Centre.

http://www.mhlw.go.jp/stf/shingi/2r9852000002xuhe-att/2r9852000002xumn.pdf

[38] Kai, J. (1996) What Worries Parents When Their Preschool Children Are Acutely Ill, Andwhy: A Qualitative Study. BMJ, 313, 983-986.

https://www.ncbi.nlm.nih.gov/pmc/articles/PMC2352339/pdf/bmj00564-0035.pdf https://doi.org/10.1136/bmj.313.7063.983

[39] Kajiyama, M. (2002) Anxiety in Parents for Children's Emergency Medicine in Japan. Japanese Society of Emergency Pediatrics, 1, 121-129.

[40] Kallestrup, P. and Bro, F. (2003) Parents' Beliefs and Expectations When Presenting with a Febrile Child at an Out-Of-Hours General Practice Clinic. British Journal of General Practice, 53, 43-44.

https://www.ncbi.nlm.nih.gov/pmc/articles/PMC1314491/pdf/12564276.pdf

[41] Shimobiraki, C. (2005) Determinants of Anxiety in Parents for Children's Emergency Medicine in Japan. Japanese Journal of Public Health, 52, 349-355. https://www.jstage.jst.go.jp/article/jph/52/4/52_349/_pdf

[42] Kushnir, T. (1984) Parental Anxiety and Children's Attendance at Emergency Departments in Relation to the Child's Birth Order. The Journal of Social Psychology, 123, 123-129. https://doi.org/10.1080/00224545.1984.9924520 
[43] E-Stat (2014) The 2010 National Census. [Cited 25 July 2017.] Available from URL: http://www.e-stat.go.jp/SG1/estat/NewList.do?tid=000001039448

[44] Kagawa Prefecture (2017) Statistics Information of Kagawa Pref. [Cited 25 July 2017.] Available from URL:

http://www.pref.kagawa.lg.jp/content/etc/subsite/toukei/index.shtml

[45] Kagawa Prefecture (2013) The Sixth Kagawa Health Care Plans. [Cited 25 July 2017.] Available from URL: http://www.qq.pref.kagawa.jp/kagawa/ap/qq/joh/pwmedplnlt01.aspx

[46] Acworth, J., Babl, F., Borland, M., Ngo, P., Krieser, D., Schutz, J., et al. (2009) Patterns of Presentation to the Australian and New Zealand Paediatric Emergency Research Network. Emergency Medicine Australasia, 21, 59-66. http://onlinelibrary.wiley.com/doi/10.1111/j.1742-6723.2009.01154.x/abstract

[47] Ban, S., Kuboyama, I., Ito, S. and Tanaka H. (2006) Time Structure of Emergency Medical Services under the Age of Twenty in Tokyo. The Journal of Japan Academy of Health Sciences, 9, 90-95.

http://mol.medicalonline.jp/library/journal/download?GoodsID=cy7healt/2006/000 902/002\&name $=0090-0095 j \& U s e r I D=133.41 .94 .76$

[48] Chishiro, T., Toshiro, J., Tsujimoto, T. and Uchino, M. (2001) The Analytical Observation of Emergency Medical Care for Children at Wakayama Medical Center. Medical Journal of Japanese Red Cross Society Wakayama Medical Center, 19, 27-33.

[49] Zimmer, K.P., Walker, A.R. and Minkovitz, C.S. (2006) Maternal and Child Factors Affecting High-Volume Pediatric Emergency Department Use. Pediatric Emergency Care, 22, 301-308.

[50] Child Care Support Section, Health and Welfare Department, Kagawa Prefecture (2017) A Medical Expense Supply System for Infants Of Kagawa Prefecture. [Cited 25 July 2017.] Available from URL:

https://kagawa-colorful.com/app-def/S-102/colorfulex/wp-content/uploads/ed4c722 f98a19f751488a5f9b315741d.pdf

[51] Gross, G.J. and Howard, M. (2001) Mothers' Decision-Making Processes regarding Health Care for Their Children. Public Health Nursing, 18, 157-168. http://onlinelibrary.wiley.com/doi/10.1046/j.1525-1446.2001.00157.x/abstract

[52] Japan Pediatric Society (2006) Pediatric Emergency. [Cited 25 July 2017.] Available from URL: http://kodomo-qq.jp/?pname $=$ n8000

[53] Maeda, K., Okamoto, S., Mishina, H. and Nakayama, T. (2009) A Decision Analysis of the Effectiveness of the Pediatric Telephone Triage Program in Japan. BioScience Trends, 3, 184-190.

http://www.biosciencetrends.com/action/downloaddoc.php?docid=245

[54] Oberlander, T.F., Pless, I.B. and Dougherty, G.E. (1993) Advice Seeking and Appropriate Use of a Pediatric Emergency Department. The American Journal of Diseases of Children, 147, 863-867.

http://jamanetwork.com/journals/jamapediatrics/article-abstract/516822

[55] Equal Employment, Children and Families Bureau, Ministry of Health, Labour and Welfare (2010) Section Meeting of Director-General, Labour Relations, Ministry of Health, Labour and Welfare.

http://www.wam.go.jp/wamappl/bb11GS20.nsf/0/24345a0348261fe9492576ac0000f d8a/\$FILE/20100115_1shiryou11_all.pdf

[56] Japan Association for the Advancement of Working Women (2009) For the Cooperation with the Emergency Support Network Service. [Cited 25 July 2017.] Availa- 
ble from URL:

http://www.jaaww.or.jp/about/pdf/document_pdf/h17_emergency_0705.pdf

[57] Sobue, I., Tanimoto, K. and Itoh, S. (2013) A Comparison of Pediatric Emergency Care on the Basis of the Number of Children and Birth Order. Japan Association for School Emergency Nursing, 15, 13-22.

[58] Gasquet, I., Villeminot, S., Estaquio, C., Durieux, P., Ravaud, P. and Falissard, B. (2004) Construction of a Questionnaire Measuring Outpatients' Opinion of Quality of Hospital Consultation Departments. Health and Quality of Life Outcomes, 2, $1-13$.

https://www.ncbi.nlm.nih.gov/pmc/articles/PMC516447/pdf/1477-7525-2-43.pdf

[59] Labarere, J., Francois, P., Auquier, P., Robert, C. and Fourny, M. (2001) Development of a French Inpatient Satisfaction Questionnaire. International Journal for Quality in Health Care, 13, 99-108.

https://academic.oup.com/intqhc/article-lookup/doi/10.1093/intqhc/13.2.99

[60] Holgado-Tello, F.P., Chacón-Moscoso, S., Barbero-García, I. and Vila-Abad, E. (2010) Polychoric versus Pearson Correlations in Exploratory and Confirmatory Factor Analysis of Ordinal Variables. Quality \& Quantity, 44, 153-166.

https://ink.springer.com/content/pdf/10.1007\%2Fs11135-008-9190-y.pdf

[61] Craney, T.A. and Surles, J.G. (2002) Model-Dependent Variance Inflation Factor Cutoff Values. Quality Engineering, 14, 391-403.

https://doi.org/10.1081/QEN-120001878

[62] Stine, R.A. (1995) Graphical Interpretation of Variance Inflation Factors. The American Statistician, 49, 53-56.

http://www.jstor.org/stable/pdf/2684812.pdf?refreqid=excelsior\%3Ad2a5f9d2bd08b a1e158763f74b101736

[63] Forero, C.G., Maydeu-Olivares, A. and Gallardo-Pujol, D. (2009) Factor Analysis with Ordinal Indicators: A Monte Carlo Study Comparing DWLS and Unweighted Least Squares Estimation. Structural Equation Modeling: A Multidisciplinary Journal, 16, 625-641.

http://www.ub.edu/gdne/amaydeusp_archivos/uls\%20vs\%20dwls\%20sem09.pdf https://doi.org/10.1080/10705510903203573

[64] Lee, C.T., Zhanga, G. and Edwards, M.C. (2012) Ordinary Least Squares Estimation of Parameters in Exploratory Factor Analysis with Ordinal Data. Multivariate Behavioral Research, 47, 314-339. https://doi.org/10.1080/00273171.2012.658340

[65] Beavers, A.S., Lounsbury, J.W., Richards, J.K., Huck, S.W., Skolits, G.J. and Esquivel, S.L. (2013) Practical Considerations for Using Exploratory Factor Analysis in Educational Research. Practical Assessment, Research \& Evaluation, 18, 1-13. http://pareonline.net/pdf/v18n6.pdf

[66] Hair, J.F., Black, B., Babin, B., Anderson, R.E. and Tatham, R.L. (2006) Factor Analysis. In: Hair, J.F., Black, B., Babin, B., Anderson, R.E. and Tatham, R.L. Eds., Multivariate Data Analysis, 6th Ed., Pearson Prentice Hall., New Jersey, 1-166.

[67] Kaiser, H.F. and Rice, J. (1974) Little Jiffy, Mark Iv. Educational and Psychological Measurement, 34, 111-117.

http://journals.sagepub.com/doi/pdf/10.1177/001316447403400115 https://doi.org/10.1177/001316447403400115

[68] Cattell, R.B. (1978) Fixing the Number of Factors: The Most Practicable Psychometric Procedures. In: Cattell, R.B., Ed., The Scientific Use of Factor Analysis in Behavioral and Life Sciences, Plenum Press, New York, 72-91.

https://doi.org/10.1007/978-1-4684-2262-7_5 
[69] Kaiser, H.F. (1960) The Application of Electronic Computers to Factor Analysis. Educational and Psychological Measurement, 20, 141-151. http://journals.sagepub.com/doi/pdf/10.1177/001316446002000116 https://doi.org/10.1177/001316446002000116

[70] Pett, M.A., Lackey, N.R. and Sullivan, J.J. (2003) Extracting the Initial Factors. In: Pett, M.A., Lackey, N.R. and Sullivan, J.J., Eds., Making Sense of Factor Analysis: The Use of Factor Analysis for Instrument Development in Health Care Research, Sage Publications, Inc., California, 114-130. https://doi.org/10.4135/9781412984898.n4

[71] Tabachnick, B.G. and Fidell, L.S. (2001) Principal Components and Factor Analysis. In: Tabachnick. B.G. and Fidell, L.S. Eds., Using Multivariate Statistics, 4th Ed., Allyn \& Bacon., Boston, 607-675.

[72] Minato, S. (1983) Some Mathematical Attitudinal Data on Eighth Grade Students in Japan Measured by a Semantic Differential. Educational Studies in Mathematics, 14, 19-38. https://link.springer.com/content/pdf/10.1007/BF00704700.pdf https://doi.org/10.1007/BF00704700

[73] Gadermann, A.M., Guhn, M. and Zumbo, B.D. (2012) Estimating Ordinal Reliability for Likert-Type and Ordinal Item Response Data: A Conceptual, Empirical, and Practical Guide. Practical Assessment, Research \& Evaluation, 17, 1-13. http://pareonline.net/pdf/v17n3.pdf

[74] Iacobucci, D. and Duhachek, A. (2003) Advancing Alpha: Measuring Reliability with Confidence. Journal of Consumer Psychology, 13, 478-487.

http://www2.owen.vanderbilt.edu/dawn.iacobucci/articles/jcpalpha_IacobucciDuha chek.pdf

[75] Zumbo, B.D., Gadermann, A.M. and Zeisser, C. (2007) Ordinal Versions of Coefficients Alpha and Theta for Likert Rating Scales. Journal of Modern Applied Statistical Methods, 6, 21-29.

http://digitalcommons.wayne.edu/cgi/viewcontent.cgi?article=1121\&context=jmasm https://doi.org/10.22237/jmasm/1177992180

[76] Helfrich, C.D., Li, Y.F., Mohr, D.C., Meterko, M. and Sales, A.E. (2007) Assessing an Organizational Culture Instrument Based on the Competing Values Framework: Exploratory and Confirmatory Factor Analyses. Implementation Science, 2, 1-14. https://implementationscience.biomedcentral.com/track/pdf/10.1186/1748-5908-213 ? site $=$ implementationscience.biomedcentral.com https://doi.org/10.1186/1748-5908-2-13

[77] Morata-Ramírez, M.A. and Holgado-Tello, F.P. (2013) Construct Validity of Likert Scales through Confirmatory Factor Analysis: A Simulation Study Comparing Different Methods of Estimation Based on Pearson and Polychoric Correlations. International Journal of Social Science Studies, 1, 54-61.

http://redfame.com/journal/index.php/ijsss/article/viewFile/27/29

[78] Yang-Wallentin, F., Jöreskoga, K.G. and Luo, H. (2010) Confirmatory Factor Analysis of Ordinal Variables with Misspecified Models. Structural Equation Modeling: A Multidisciplinary Journal, 17, 392-423. https://doi.org/10.1080/10705511.2010.489003

[79] Hooper, D., Coughlan, J. and Mullen, M. (2008) Structural Equation Modelling: Guidelines for Determining Model Fit. The Electronic Journal of Business Research Methods, 6, 53-60.

http://arrow.dit.ie/cgi/viewcontent.cgi?article=1001\&context=buschmanart

[80] Schermelleh-Engel, K., Moosbrugger, H. and Müller, H. (2003) Evaluating the Fit of 
Structural Equation Models: Tests of Significance and Descriptive Goodness-Of-Fit Measures. Methods of Psychological Research Online, 8, 23-74.

http://www.stats.ox.ac.uk/ snijders/mpr_Schermelleh.pdf

[81] Sin, S.J. (2012) Modeling the Impact of Individuals' Characteristics and Library Service Levels on High School Students' Public Library Usage: A National Analysis. Library \& Information Science Research, 34, 228-237.

http://www.sciencedirect.com/science/article/pii/S0740818812000333

[82] Chin, W.W. (1998) Issues and Opinion on Structural Equation Modeling. MIS Quarterly, 22, 7-16.

https://www.jstor.org/stable/pdf/249674.pdf?refreqid=excelsior\%3Ac8b2bd4af35e73 0c294bf94a088d8290

[83] Hair, J.F., Black, B., Babin, B., Anderson, R.E. and Tatham, R.L. (2006) SEM: Confirmatory Factor Analysis. In: Hair, J.F., Black, B., Babin, B., Anderson, R.E. and Tatham, R.L. Eds., Multivariate Data Analysis, 6th Ed., Pearson Prentice Hall, New Jersey, 770-836.

[84] Starkweather, J. (2012) Step Out of the Past: Stop Using Coefficient Alpha; There Are Better Ways to Calculate Reliability. Research and Statistical Support, 1-6. https://it.unt.edu/sites/default/files/omega_jds_jun2012.pdf

[85] Thurber, S. and Bonynge, M.R. (2011) SEM-Based Composite Reliability Estimates of the Crisis Acuity Rating Scale with Children and Adolescents. Archives of Assessment Psychology, 1, 1-9.

http://assessmentpsychologyboard.org/journal/index.php/AAP/article/viewFile/27/ $\underline{13}$

[86] Fornell, C. and Larker, D.F. (1981) Evaluating Structural Equation Models with Unobservable Variables and Measurement Error. Journal of Marketing Research, 18, 39-50. https://doi.org/10.2307/3151312

[87] Kalpakjian, C.Z., Toussaint, L.L., Albright, K.J., Bombardier, C.H., Krause, J.K. and Tate, D.G. (2009) Patient Health Questionnaire-9 in Spinal Cord Injury: An Examination of Factor Structure as Related to Gender. The Journal of Spinal Cord Medicine, 32, 147-156.

https://www.ncbi.nlm.nih.gov/pmc/articles/PMC2678286/pdf/i1079-0268-32-2-147. pdf https://doi.org/10.1080/10790268.2009.11760766

[88] Lorenzo-Seva, U. and ten Berge, J.M.F. (2006) Tucker's Congruence Coefficient as a Meaningful Index of Factor Similarity. Methodology, 2, 57-64.

https://openpsych.net/forum/attachment.php?aid=364 https://doi.org/10.1027/1614-2241.2.2.57

[89] Milfont, T.L. and Fischer, R. (2010) Testing Measurement Invariance across Groups: Applications in Cross-Cultural Research. International Journal of Psychological Research, 3, 111-121.

[90] Wu, A.D., Li, Z. and Zumbo, B.D. (2007) Decoding the Meaning of Factorial Invariance and Updating the Practice of Multi-Group Confirmatory Factor Analysis: A Demonstration with TIMSS Data. Practical Assessment, Research \& Evaluation, 12 , 1-26. http://pareonline.net/getvn.asp? $\mathrm{v}=12 \& \mathrm{n}=3$

[91] Breslow, N.E. (1996) Statistics in Epidemiology: The Case-Control Study. Journal of the American Statistical Association, 91, 14-28.

http://personal.psc.isr.umich.edu/yuxie-web/files/pubs/Articles/Breslow1996.pdf https://doi.org/10.1080/01621459.1996.10476660

[92] Lachin, J.M. (2011) Biostatistical Methods: The Assessment of Relative Risks, 2nd 
Edition, Wiley, New Jersey.

[93] LaMorte, W.W. (2016) The Cochran-Mantel-Haenszel Method. [Cited 25 July 2017.] Available from URL:

http://sphweb.bumc.bu.edu/otlt/MPH-Modules/BS/BS704-EP713_Confounding-E M/BS704-EP713_Confounding-EM7.html

[94] McCulloch, C.E. (2000) Generalized Linear Models. Journal of the American Statistical Association, 95, 1320-1324.

http://amstat.tandfonline.com/doi/pdf/10.1080/01621459.2000.10474340?needAcces $\underline{s=\text { true }}$ https://doi.org/10.1080/01621459.2000.10474340

[95] Olsson, U. (2002) Generalized Linear Models: An Applied Approach. Student Litteratur Lund., Sweden, 1-232.

ftp://nozdr.ru/biblio/kolxo3/M/MV/MVsa/Olsson\%20U.\%20Generalized\%20linear \%20models.\%20An\%20applied\%20approach\%20(Studentlitteratur,\%202002)(ISBN \%209144041551)(O)(243s)_MVsa_.pdf

[96] Abdi, H. and Valentin, D. (2007) Multiple Correspondence Analysis. In: Salkind, N.J., Ed., Encyclopedia of Measurement and Statistics, SAGE Publications, Thousand Oaks, CA, 1-13.

[97] Hoffman, D.L. and Leeuw, J.D. (1992) Interpreting Multiple Correspondence Analysis as a Multidimensional Scaling Method. Marketing Letters, 3, 259-272.

https://link.springer.com/content/pdf/10.1007\%2FBF00994134.pdf https://doi.org/10.1007/BF00994134

[98] Cabinet Office, Government of Japan (2015) Local Support Programs for Children and Child-Rearing.

http://www8.cao.go.jp/shoushi/shinseido/administer/setsumeikai/h270123/pdf/s3-1 .pdf

[99] E-Stat (2011) The 2010 National Census. [Cited 25 July 2017.] Available from URL: http://www.e-stat.go.jp/SG1/estat/List.do?bid=000001034991\&cycode $=0$

[100] Mori, R., Etani, Y., Ehara, A., Oyama, S., Nagamura, T., Kajii, N., et al. (2015) Report of System to Provide Pediatric Medicine. The Journal of the Japan Pediatric Society, 119, 1551-1566. 
Appendix 1. Polychoric Correlation Matrix (Odd Number Group, N = 7,255)

\begin{tabular}{|c|c|c|c|}
\hline Number & Item & 1 & 2 \\
\hline 1 & Obtain information on the facilities providing consultation at night and on holidays. & & \\
\hline 2 & Judge the disease state and take action via the Internet or by cellular phone. & 0.56 & \\
\hline 3 & Use telephone consultation. & 0.48 & 0.70 \\
\hline 4 & Use facilities for nurturing diseased and convalescent children. & 0.33 & 0.48 \\
\hline 5 & Use Kagawa Emergency Support Network. & 0.38 & 0.56 \\
\hline 6 & Obtain knowledge on childhood diseases and injuries. & 0.50 & 0.49 \\
\hline 7 & Judge the state of your child's diseases and injuries by yourself. & 0.28 & 0.39 \\
\hline 8 & Take care of your child's diseases and injuries by yourself. & 0.32 & 0.33 \\
\hline 9 & $\begin{array}{l}\text { There is no family member or friend with whom you can consult } \\
\text { about your child's diseases and injuries. }\end{array}$ & 0.18 & 0.21 \\
\hline 10 & There is no family member or friend whom you can ask to take care of your child. & 0.14 & 0.15 \\
\hline 11 & There is no facility for nurturing diseased and convalescent children nearby. & 0.27 & 0.28 \\
\hline 12 & There is no branch of the Kagawa Emergency Support Network nearby. & 0.34 & 0.37 \\
\hline 13 & There is no facility providing emergency consultation in the daytime or on holidays. & 0.30 & 0.27 \\
\hline 14 & There is no facility providing consultation at night ( 5 p.m. to around 11 p.m.). & 0.30 & 0.25 \\
\hline 15 & $\begin{array}{l}\text { There is no facility providing overnight consultation } \\
\text { (from around } 11 \text { p.m. to } 9 \text { a.m. the next morning). }\end{array}$ & 0.30 & 0.28 \\
\hline 16 & There is no facility providing consultation at any time. & 0.32 & 0.29 \\
\hline 17 & Emergency facilities available change day by day. & 0.42 & 0.35 \\
\hline 18 & The locations of emergency facilities available are unclear. & 0.34 & 0.31 \\
\hline 19 & It takes over 30 minutes from home to get to an emergency facility providing consultation. & 0.30 & 0.27 \\
\hline 20 & Consultation is not always performed by a pediatrician. & 0.28 & 0.29 \\
\hline 21 & The doctor is not your personal doctor. & 0.35 & 0.31 \\
\hline 22 & Waiting time is long. & 0.25 & 0.26 \\
\hline 23 & Examinations including X-ray, CT, or blood or urine tests are not available. & 0.22 & 0.28 \\
\hline 24 & Treatments including IV, injection, or inhalation are not available. & 0.20 & 0.25 \\
\hline 25 & No medicines for internal use, such as an antipyretic or antibiotic, are prescribed. & 0.20 & 0.22 \\
\hline 26 & Pain and symptoms are not relieved appropriately. & 0.17 & 0.22 \\
\hline 27 & In the emergency consultation hospital, there is no facility to stay at. & 0.21 & 0.23 \\
\hline 28 & There is no support for a parent's insufficient experience/knowledge, anxiety, and concerns. & 0.23 & 0.27 \\
\hline 29 & There is no reasonable explanation of the disease and treatment. & 0.16 & 0.21 \\
\hline 30 & $\begin{array}{l}\text { What you should be careful about at home, such as how to } \\
\text { administer medicines and meals, is not explained. }\end{array}$ & 0.18 & 0.21 \\
\hline 31 & No indication regarding the patient's next consultation is given. & 0.21 & 0.23 \\
\hline
\end{tabular}




\begin{tabular}{|c|c|c|c|c|c|c|c|c|c|c|c|c|c|c|c|c|c|}
\hline $\mathbf{N}$ & 3 & 4 & 5 & 6 & 7 & 8 & 9 & 10 & 11 & 12 & 13 & 14 & 15 & 16 & 17 & 18 & 19 \\
\hline 4 & 0.59 & & & & & & & & & & & & & & & & \\
\hline 5 & 0.68 & 0.75 & & & & & & & & & & & & & & & \\
\hline 6 & 0.47 & 0.38 & 0.47 & & & & & & & & & & & & & & \\
\hline 7 & 0.32 & 0.25 & 0.31 & 0.52 & & & & & & & & & & & & & \\
\hline 8 & 0.31 & 0.23 & 0.27 & 0.47 & 0.72 & & & & & & & & & & & & \\
\hline 9 & 0.21 & 0.15 & 0.18 & 0.23 & 0.32 & 0.41 & & & & & & & & & & & \\
\hline 10 & 0.16 & 0.14 & 0.17 & 0.17 & 0.26 & 0.27 & 0.84 & & & & & & & & & & \\
\hline 11 & 0.30 & 0.36 & 0.33 & 0.27 & 0.29 & 0.31 & 0.43 & 0.47 & & & & & & & & & \\
\hline 12 & 0.40 & 0.38 & 0.45 & 0.34 & 0.33 & 0.34 & 0.38 & 0.40 & 0.75 & & & & & & & & \\
\hline 13 & 0.24 & 0.20 & 0.22 & 0.24 & 0.30 & 0.27 & 0.55 & 0.50 & 0.46 & 0.52 & & & & & & & \\
\hline 14 & 0.22 & 0.16 & 0.18 & 0.20 & 0.28 & 0.25 & 0.56 & 0.51 & 0.43 & 0.46 & 0.86 & & & & & & \\
\hline 15 & 0.23 & 0.19 & 0.22 & 0.22 & 0.32 & 0.25 & 0.48 & 0.45 & 0.41 & 0.46 & 0.82 & 0.87 & & & & & \\
\hline 16 & 0.23 & 0.19 & 0.22 & 0.22 & 0.31 & 0.26 & 0.44 & 0.42 & 0.41 & 0.46 & 0.78 & 0.82 & 0.93 & & & & \\
\hline 17 & 0.33 & 0.29 & 0.32 & 0.32 & 0.33 & 0.32 & 0.31 & 0.29 & 0.39 & 0.46 & 0.56 & 0.57 & 0.63 & 0.67 & & & \\
\hline 18 & 0.27 & 0.22 & 0.25 & 0.26 & 0.33 & 0.29 & 0.44 & 0.41 & 0.39 & 0.45 & 0.63 & 0.64 & 0.65 & 0.67 & 0.75 & & \\
\hline 19 & 0.22 & 0.19 & 0.22 & 0.23 & 0.29 & 0.26 & 0.40 & 0.38 & 0.40 & 0.43 & 0.64 & 0.66 & 0.66 & 0.66 & 0.63 & 0.72 & \\
\hline 20 & 0.24 & 0.22 & 0.25 & 0.22 & 0.31 & 0.25 & 0.28 & 0.28 & 0.32 & 0.38 & 0.50 & 0.51 & 0.54 & 0.55 & 0.59 & 0.58 & 0.62 \\
\hline 21 & 0.30 & 0.30 & 0.30 & 0.31 & 0.28 & 0.30 & 0.17 & 0.16 & 0.31 & 0.39 & 0.31 & 0.29 & 0.32 & 0.36 & 0.54 & 0.41 & 0.44 \\
\hline 22 & 0.24 & 0.26 & 0.25 & 0.26 & 0.31 & 0.27 & 0.17 & 0.19 & 0.28 & 0.34 & 0.33 & 0.30 & 0.35 & 0.37 & 0.46 & 0.42 & 0.45 \\
\hline 23 & 0.25 & 0.22 & 0.24 & 0.22 & 0.30 & 0.26 & 0.32 & 0.31 & 0.31 & 0.35 & 0.48 & 0.48 & 0.49 & 0.51 & 0.49 & 0.52 & 0.55 \\
\hline 24 & 0.21 & 0.20 & 0.22 & 0.19 & 0.30 & 0.25 & 0.36 & 0.34 & 0.32 & 0.34 & 0.52 & 0.52 & 0.53 & 0.53 & 0.47 & 0.53 & 0.56 \\
\hline 25 & 0.19 & 0.17 & 0.19 & 0.17 & 0.29 & 0.24 & 0.36 & 0.33 & 0.30 & 0.30 & 0.50 & 0.51 & 0.51 & 0.50 & 0.44 & 0.51 & 0.53 \\
\hline 26 & 0.18 & 0.16 & 0.19 & 0.17 & 0.32 & 0.25 & 0.41 & 0.37 & 0.29 & 0.30 & 0.53 & 0.56 & 0.56 & 0.54 & 0.44 & 0.53 & 0.56 \\
\hline 27 & 0.19 & 0.17 & 0.19 & 0.18 & 0.29 & 0.23 & 0.37 & 0.34 & 0.30 & 0.33 & 0.54 & 0.55 & 0.57 & 0.58 & 0.50 & 0.54 & 0.58 \\
\hline 28 & 0.23 & 0.21 & 0.24 & 0.26 & 0.40 & 0.33 & 0.42 & 0.37 & 0.32 & 0.36 & 0.49 & 0.51 & 0.51 & 0.51 & 0.46 & 0.52 & 0.52 \\
\hline 29 & 0.17 & 0.16 & 0.17 & 0.16 & 0.32 & 0.27 & 0.42 & 0.37 & 0.28 & 0.28 & 0.51 & 0.54 & 0.53 & 0.52 & 0.41 & 0.51 & 0.53 \\
\hline 30 & 0.18 & 0.17 & 0.19 & 0.19 & 0.33 & 0.27 & 0.41 & 0.35 & 0.29 & 0.29 & 0.47 & 0.49 & 0.48 & 0.47 & 0.39 & 0.48 & 0.50 \\
\hline 31 & 0.20 & 0.18 & 0.21 & 0.20 & 0.31 & 0.28 & 0.38 & 0.33 & 0.29 & 0.30 & 0.45 & 0.46 & 0.45 & 0.45 & 0.41 & 0.48 & 0.49 \\
\hline $\mathbf{N}$ & \multicolumn{2}{|c|}{20} & 21 & \multicolumn{2}{|l|}{22} & 23 & 24 & \multicolumn{2}{|l|}{25} & 26 & 27 & \multicolumn{2}{|c|}{28} & 29 & \multicolumn{2}{|c|}{30} & 31 \\
\hline 21 & & & & & & & & & & & & & & & & & \\
\hline 22 & & & 0.57 & & & & & & & & & & & & & & \\
\hline 23 & & 52 & 0.52 & 0.60 & & & & & & & & & & & & & \\
\hline 24 & & 52 & 0.46 & 0.55 & & 0.92 & & & & & & & & & & & \\
\hline 25 & & 58 & 0.41 & 0.48 & & 0.79 & 0.87 & & & & & & & & & & \\
\hline 26 & & & 0.39 & 0.49 & & 0.78 & 0.86 & 0.89 & & & & & & & & & \\
\hline 27 & & 57 & 0.39 & 0.47 & & 0.72 & 0.77 & 0.77 & & 0.82 & & & & & & & \\
\hline 28 & & & 0.44 & 0.51 & & 0.67 & 0.70 & 0.69 & & 0.77 & 0.74 & & & & & & \\
\hline 29 & & & 0.35 & 0.46 & & 0.67 & 0.73 & 0.72 & & 0.82 & 0.75 & & .85 & & & & \\
\hline 30 & & & 0.34 & 0.44 & & 0.64 & 0.69 & 0.70 & & 0.78 & 0.72 & & .81 & 0.90 & & & \\
\hline 31 & & & 0.37 & 0.46 & & 0.66 & 0.69 & 0.70 & & 0.76 & 0.71 & & .79 & 0.86 & 0.9 & & \\
\hline
\end{tabular}

The Kagawa Emergency Support Network is a contract system in which parents ask trained volunteers to take care of their ill children. A VIF value of more than five (item number, VIF; $15 / 16,7.66 ; 23 / 24,6.33 ; 25 / 26,5.02 ; 29 / 30,5.33 ; 30 / 31,5.75)$ and four items $(15,24,25$, and 30$)$ were omitted from the polychoric correlation matrix in order to exclude multicollinearity. N: Number. 
Submit or recommend next manuscript to SCIRP and we will provide best service for you:

Accepting pre-submission inquiries through Email, Facebook, LinkedIn, Twitter, etc. A wide selection of journals (inclusive of 9 subjects, more than 200 journals)

Providing 24-hour high-quality service

User-friendly online submission system

Fair and swift peer-review system

Efficient typesetting and proofreading procedure

Display of the result of downloads and visits, as well as the number of cited articles Maximum dissemination of your research work

Submit your manuscript at: http://papersubmission.scirp.org/

Or contact health@scirp.org 